\title{
REDES MOLECULARES: UMA ANÁLISE SOBRE ANOTAÇÕES E DESCOBERTA DE NOVOS ATIVOS
}

\author{
Alan C. Pilon ${ }^{a, b}$, Natália C. Vieira ${ }^{a}$, Juliano G. Amaral ${ }^{\mathrm{c}}$, Afif F. Monteiro ${ }^{\mathrm{d}}$, Ricardo R. da Silva ${ }^{\mathrm{b}}$, Laila S. Spíndola ${ }^{\mathrm{e}}$ Ian \\ Castro-Gamboa ${ }^{a}$ e Norberto P. Lopes ${ }^{b, *}$, (1) \\ a'Departamento de Química Orgânica, Instituto de Química, Universidade Estadual Paulista, 14800-060 Araraquara - SP, Brasil \\ bDepartamento de Ciências Biomoleculares, Faculdade de Ciências Farmacêuticas, Universidade de São Paulo, 14040-903 Ribeirão \\ Preto - SP, Brasil \\ 'Instituto Multidisciplinar em Saúde, Campus Anísio Teixeira, Universidade Federal da Bahia, 45029-094 Vitória da Conquista \\ - BA, Brasil \\ 'Instituto de Química de São Carlos, Universidade de São Paulo, 13560-970 São Carlos - SP, Brasil \\ eUniversidade de Brasília, Campus Universitário Darcy Ribeiro, 70910-900 Brasília - DF, Brasil
}

Recebido em 25/03/2021; aceito em 22/04/2021; publicado na web em 15/06/2021

\begin{abstract}
MOLECULAR NETWORKS: AN ANALYSIS ON ANNOTATIONS AND DISCOVERY OF NEW ASSETS. To speed up the discovery of bioactive natural products (NP), chemists have sought advanced approaches in analytical and computational chemistry in attempt to organize and extract information from large data sets. In this sense, the molecular networks (MN) successfully organized enormous sets of mass spectrometry (MS) data together with samples metadata information in an intuitive visualization in the spectral similarity networks format. GNPS (Global Natural Products Social Molecular Networking), a free online platform for storing and processing $\mathrm{MS}^{\mathrm{n}}$ data, is a leading application of spectral matching with public databases aimed at the dereplication and discovery of new bioactive products through molecular networks. In this review, we address the concept of GNPS spectral similarity networks, as well as their complementary computational tools, benefits and limitations applied in NP studies associated with dereplication, chemical ecology, functional genetics and determination of biosynthetic pathways.
\end{abstract}

Keywords: natural products; tandem mass spectrometry; molecular networking; dereplication; chemical ecology.

\section{INTRODUÇÃO}

A busca por procedimentos ágeis e eficientes para descoberta de novos produtos naturais bioativos impulsionou, no final dos anos de 90, o avanço das tecnologias analíticas em separação e aquisição espectral. ${ }^{1} \mathrm{O}$ desenvolvimento de ferramentas computacionais, como as técnicas de elucidação de estrutura assistida por computador (CASE - do inglês Computer Assisted Structure Elucidation), permitiu também acelerar a identificação de compostos por meio de comparações individuais entre espectros adquiridos e repositórios espectrais de compostos. ${ }^{2}$

Embora essa comparação individual entre espectros tenha contribuído para anotação e desreplicação de muitas substâncias, o procedimento continua sendo laborioso, demandando longos períodos de curagem e verificação dos dados. ${ }^{3,4}$ Se consideramos as abordagens modernas aplicadas aos produtos naturais, como metabolômica ${ }^{5,6}$ ou a biologia de sistemas, a situação fica ainda mais complexa e impraticável. Plantas, microrganismos (terrestres e marinhos) e bactérias podem expressar, dependendo das condições ambientais e genéticas, centenas ou até milhares de compostos, tornando a tarefa de identificar/anotar compostos por meio das estratégias tradicionais em PN um desafio humanamente inviável. ${ }^{1,7}$

A química de produtos naturais voltada as ciências ômicas, ou ao entendimento de eventos multifatoriais, tem buscado na análise estatística, bioinformática e na quimiometria formas alternativas de organizar, armazenar, mapear e analisar conjuntos complexos de dados, com rapidez e confiabilidade. ${ }^{8}$ Análise por redes moleculares (do inglês Molecular Networking) surge neste contexto como uma ferramenta de organização e visualização de grandes conjuntos de dados. ${ }^{9-12}$ A partir de dados espectrais, a rede molecular facilita a identificação de padrões

*e-mail: npelopes@gmail.com (similaridades e diferenças espectrais) entre conjuntos amostrais. Isso permite reconhecer classes metabólicas, substituintes e funções orgânicas em estruturas complexas de produtos naturais. ${ }^{13-28}$

A incorporação de bibliotecas de compostos (espectrais) nas redes moleculares têm auxiliado a anotação de compostos conhecidos por comparação direta (dado adquirido e as bibliotecas) mas também tem fornecido subsídios para anotação de moléculas desconhecidas. ${ }^{3,9} \mathrm{O}$ aumento em número e quantidade de dados presentes em repositórios espectrais, como por exemplo o PubChem, ${ }^{29}$ METLIN, ${ }^{30}$ NIST, ${ }^{31}$ GOLM, ${ }^{32}$ BMRB, ${ }^{33}$ Super Natural $\mathrm{II}^{32}$ e a $\mathrm{NuBBE}_{\mathrm{DB}}{ }^{34}$ também tem ajudado no desenvolvimento de abordagens computacionais complementares as redes moleculares.

A Global Natural Products Social Networking ${ }^{3}$ (GNPS) é uma plataforma online e de livre acesso para o cálculo de redes moleculares a partir de dados de espectrometria de massas. ${ }^{3}$ Essa plataforma, inicialmente desenvolvida para estudos proteômicos, ${ }^{35}$ foi, a princípio, adaptada para análise metabólica de organismos marinhos e atualmente é utilizada por toda comunidade de PN. ${ }^{36}$ Sua eficiência em organizar e resumir grandes conjuntos de dados de espectrometria de massas (EM) levaram a expansão de seu protótipo de aplicação em linha de comando para uma abordagem de aplicação web, com uma interface simples e amigável a não programadores. A infraestrutura computacional do Mass spectrometry Virtual Interactive Environment (MassIVE), um repositório de dados comunitário, parte do consórcio global ProteomeXchange, foi utilizado como suporte para criação do GNPS, o que transformou esta plataforma em uma rede internacional de tratamento e visualização de grandes conjuntos de dados. ${ }^{13,37,38}$ É importante destacar que plataformas como o GNPS podem ser de grande utilidade para a anotação espectral, o que não necessariamente significa identificação estrutural. ${ }^{39}$

O objetivo deste trabalho é descrever como as redes moleculares, a partir da plataforma GNPS, tem impactado no desenvolvimento 
da química de produtos naturais do século XXI. Esta revisão traz informações sobre a criação das redes moleculares e uso das diferentes ferramentas associadas, como também exemplos interdisciplinares de aplicação em produtos naturais.

\section{CONCEITOS QUE ENVOLVEM AS REDES MOLECULARES}

O conceito de redes pode ser aplicado a qualquer campo acadêmico interdisciplinar no estudo de dados complexos. Seu primeiro relato é descrito por Leonhard Euler em 1736 no caso matemático das Sete Pontes de Königsberg. ${ }^{40}$ As redes moleculares por sua vez visam, através de uma estratégia computacional, ajudar a visualização e interpretação de dados espectrais complexos. Utilizando de fatores correlacionais, como por exemplo o grau de similaridade entre espectros, é possível gerar redes moleculares que, em última análise, podem ajudar na determinação de classes metabólicas, funções orgânicas, propriedades biológicas e quimiotaxonômicas. ${ }^{8,11}$

A plataforma GNPS ${ }^{3}$ é voltada à análise de grandes conjuntos de dados (a partir de íons fragmento) em experimentos de espectrometria de massas. Esses dados podem ser gerados a partir de aquisição dependente de dados (DDA), onde os experimentos de $\mathrm{EM}^{\mathrm{n}}$ são realizados pelo isolamento prévio de íon(s) ou, pela aquisição independente de dados (DIA), em que os espectros de $\mathrm{EM}^{\mathrm{n}}$ são adquiridos sem isolamento prévios dos íons precursores. Diversas fontes de ionização podem ser utilizadas para a criação das redes moleculares, como ESI, APCI, MALDI e EI, bem como diversas fontes fragmentação, como collision induced dissociation (CID), Higher-energy collisional dissociation (HCD) e Electron-transfer dissociation (ETD). Contudo, as informações da EM de uma substância podem variar significativamente dependendo da técnica de ionização $0^{41}$ ou da forma de ativação colisional, ${ }^{42}$ o que impossibilita o uso de diferentes protocolos/instrumentação para análise de um conjunto de amostras.

O conjunto de algoritmos do GNPS organiza cada espectro adquirido (por exemplo em CLAE-EM e $E^{\mathrm{n}}$ ) e os classifica a partir de um valor similaridade, que nesse caso é calculado pelo valor de cosseno entre espectros presentes na(s) amostra(s) ou entre $\mathrm{a}(\mathrm{s}) \operatorname{amostra}(\mathrm{s})$ e o(s) espectro(s) de referência presentes nos bancos de dados. Dessa forma, são gerados agrupamentos espectrais com base em um valor pré-estabelecido de cosseno (valor que varia de 0 a 1 - sendo que 1 indica $100 \%$ similaridade ou identidade e 0 , totalmente diferentes). Se o pareamento entre dois espectros possuir uma similaridade de cosseno maior ou igual ao valor limite préestabelecido pelo usuário, uma conexão ou um arco entre os dois espectros é estabelecido. ${ }^{3,10}$
Como cada substância pode ser constituída por um conjunto de espectros (em experimentos CLAE-EM e EM ${ }^{\mathrm{n}}$ podem ocorrer várias fragmentações de uma mesma substância devido a largura do sinal cromatográfico) é necessária uma etapa de agrupamento de íons de uma mesma substância, também chamado de espectro consenso. Esse espectro é representado por um nodo (todos os espectros de uma mesma substância ficam armazenados neste nodo) enquanto os arcos que os conectam representam os valores de similaridade entre os nodos. ${ }^{12}$

Finalmente, os nodos da rede são anotados utilizando diferentes bibliotecas de compostos, com os mesmos critérios de pareamento espectral, número mínimo de picos compartilhados (no espectro de massas) e a maximização da similaridade do cosseno, considerando a diferença das massas entre os íons precursores. ${ }^{3,10,12}$

A Figura 1 representa o processo de construção das redes moleculares a partir de dados de CLAE-EM e EM ${ }^{\mathrm{n}}$. São extraídos dos sinais (cromatográficos) os espectros de $\mathrm{EM}^{\mathrm{n}}$ comparados, formando os espectros consenso e a rede molecular a partir de um valor préestabelecido de similaridade de cosseno.

A plataforma também oferece armazenamento e análise de dados brutos (cromatogramas completos em formatos públicos $m z M L \mathrm{e}$ $m z X M L$ ) através do repositório de dados MassIVE, possibilitando o compartilhamento com toda a comunidade. ${ }^{3}$ Após a geração dos nodos e arcos, estes são representados como uma rede, Figura 1, permitindo que a informação química seja interpretada de forma intuitiva, associando compostos análogos, e suas respectivas particularidades químicas (diferenças de massas associadas aos arcos) sejam agrupadas em uma representação condensada.

A Figura 2 representa a estrutura da plataforma de redes moleculares (GNPS), considerando desde o carregamento dos dados, armazenamento, rede social e comunitária da plataforma como as ferramentas de análise. Como a plataforma está constantemente disponibilizando novas ferramentas, os usuários são aconselhados a verificar o fórum oficial da comunidade para eventuais dúvidas (https://groups.google. com/forum/\#!forum/molecular_networking_bug_reports).

\section{REDES MOLECULARES - MODO CLÁSSICO GNPS (CMN)}

A rede molecular clássica, conhecida como Classic Molecular Networking no GNPS, foi a primeira ferramenta desenvolvida na plataforma dispensando a necessidade de estágios de préprocessamento (avançado) como a quantificação dos features. Neste modo é possível carregar dados de CLAE ou CLUE-EM e EM ${ }^{\mathrm{n}}$ e MALDI-EM em modo DDA. ${ }^{3,10,43}$

A criação das redes moleculares começa pelo processo de remoção de ruídos dos espectros de fragmentação $\left(\mathrm{EM}^{\mathrm{n}}\right)$, com a seleção dos

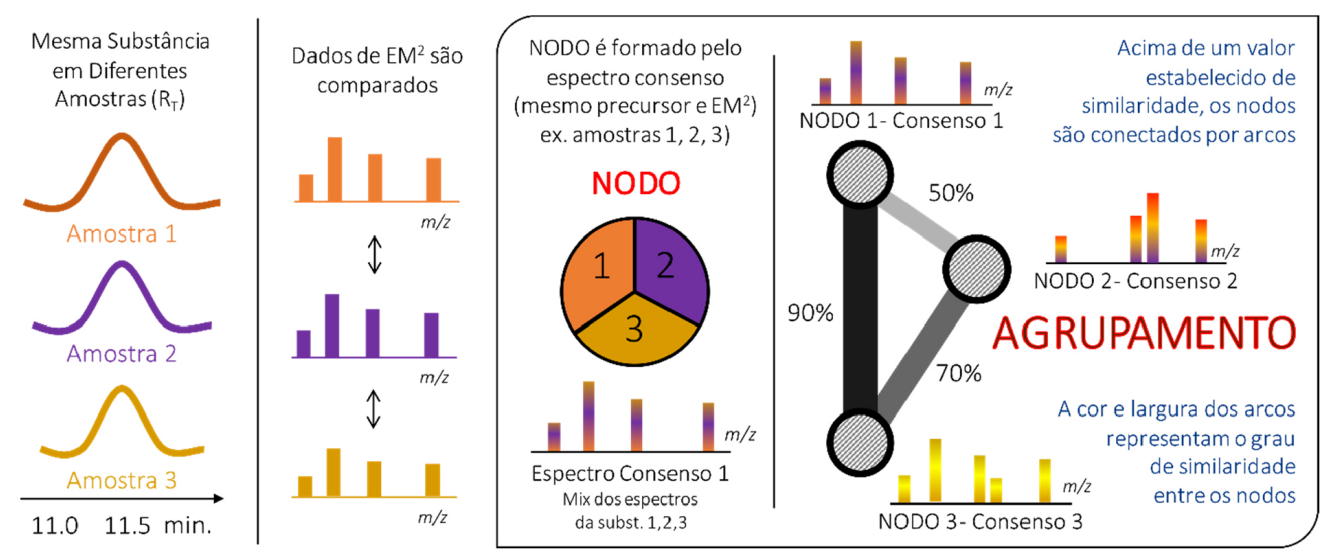

Figura 1. Formação de uma rede molecular a partir da inserção dos dados de EM"n comparação dos espectros de massas para a geração do espectro de consenso e formação de agrupamento por similaridade (clusters) 


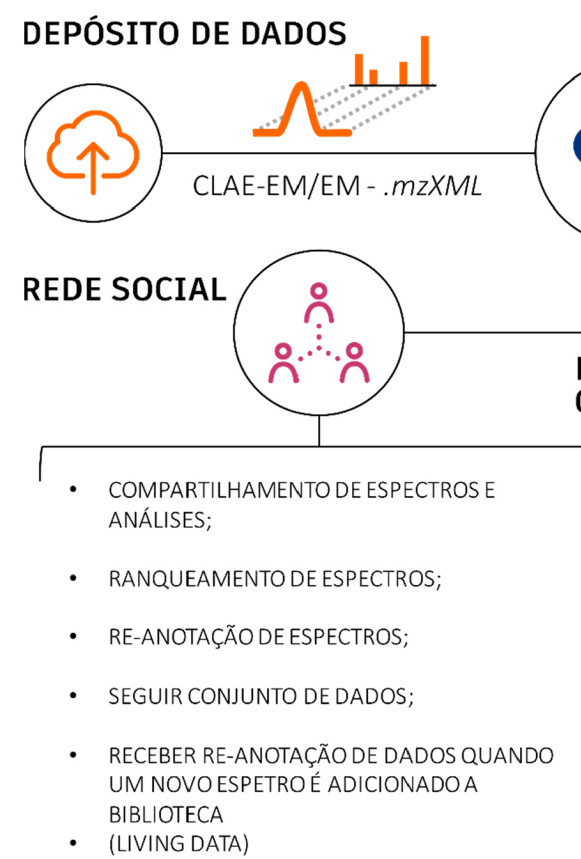

MassiVE $\mid \begin{aligned} & \text { MASS SPECTROMETRY INTERACTIVE } \\ & \text { VIRTUAL ENVIRONMENT }\end{aligned}$

ARMAZENAMENTO DADOS

BIBLIOTECA ESPECTRALDE REFERÊNCIA

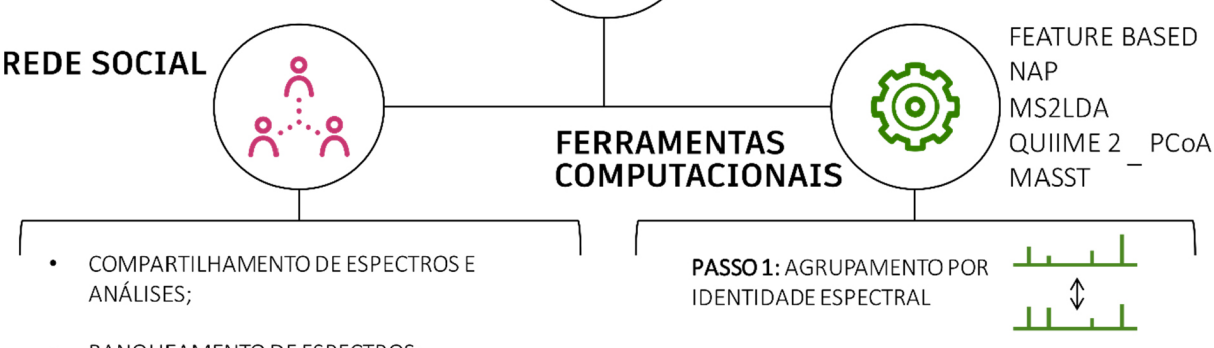

PASSO 2: SIMILARIDADE ESPECTRAL ENTRE GRUPOS

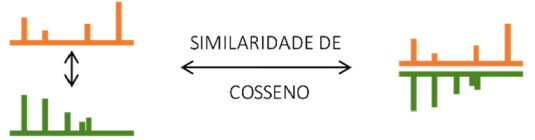

PASSO 3: FORMAÇÃO DA REDE MOLECULARE PAREAMENTO ESPECTRAL COM AS BIBLIOTECAS

Figura 2. Representação esquemática da estrutura da plataforma GNPS. A plataforma conta com duas bases de dados, que armazenam dados brutos experimentais e dados de referência. Além das bases de dados existe um componente analítico, que está em constante expansão, com a adição de novos módulos e um componente social, onde os usuários podem trocar informações, depositar e analisar dados

$N$ picos mais intensos (parâmetro definido pelo usuário) em janelas de $+/-50$ unidades de $\mathrm{m} / \mathrm{z}$ ao longo do espectro. Em seguida, para a criação dos espectros consenso (espectros pertencentes a um único sinal cromatográfico), o GNPS oferece o algoritmo MSCluster, ${ }^{44} \mathrm{o}$ qual é possível ajustar a tolerância de massa para agrupamento de espectros referentes ao íon precursor e aos íons produto (valor padrão de 1.0 Da). Esses valores dependem da performance do instrumento de massas (alta ou baixa resolução). Assim, equipamentos calibrados com maior poder de resolução utilizam tolerâncias menores que 1.0 Da, como por exemplo instrumentos com analisadores do tipo Time of Flight (ToF) ou Orbitrap.

A vantagem desta abordagem é que ela requer um número menor de etapas de pré-processamento de dados, pode ser utilizada em pequenos e grandes conjuntos de amostras e não necessita de um protocolo de quantificação para as análises experimentais. A desvantagem é que o algoritmo MSCluster não leva em consideração o tempo de retenção dos sinais, o que pode levar ao agrupamento de isômeros e isóbaros que possuem algum nível de similaridade espectral. Ele também não é indicado para a criação de redes que buscam identificar flutuações na quantidade e na covariância dos compostos da rede. ${ }^{45,46}$

Uma das primeiras publicações em produtos naturais utilizando de redes moleculares foi desenvolvido por Watrous e colaboradores, ${ }^{12}$ trabalhando com entendimento de interações de colônias de bactérias. O potencial da técnica foi explorado para detecção, visualização e classificação química dos compostos.

Dentre as possíveis aplicações em produtos naturais do modo clássico destacam-se a anotação de compostos com eficiência e rapidez, estudos de quimiotaxonomia e descoberta de novas substâncias. ${ }^{18,37,47-49}$ Como as redes moleculares formam agrupamentos por similaridade espectral, muitas substâncias de uma mesma classe metabólica (perfil de fragmentação similar) podem ser arranjadas dentro de um mesmo agrupamento. Isso é bastante vantajoso quando se analisa grandes conjuntos amostrais ( $>15$ amostras), pois a rede permite uma avaliação simultânea de todos os espectros da rede.

É possível ainda utilizar o banco de dados para aumentar anotação de várias classes metabólicas, priorizando compostos inéditos que possuem similaridade espectral com compostos conhecidos. Esse procedimento é chamado de identificação de desconhecidos por compostos conhecidos, uma estratégia útil e rápida para a descoberta de novos produtos naturais. Se os compostos conhecidos de interesse possuem informações sobre alguma atividade biológica, essa priorização pode carregar novas informações biológicas a um dado organismo. O trabalho pioneiro de Yang e colaboradore ${ }^{11}$ utilizando de redes moleculares para o estudo de microrganismos marinhos e terrestres, desreplicou mais 58 moléculas bioativas a partir de análogos dos bancos de dados, abrindo espaço para o crescimento dessa estratégia.

\section{REDES MOLECULARES - MODO FEATURE BASED MOLECULAR NETWORKING GNPS (FBMN)}

Para contornar os problemas causados pelo uso do algoritmo MSCluster e, também considerar a flutuação e quantificação metabólica, o GNPS disponibilizou um módulo de análise chamado de Feature-Based Molecular Networking (FBMN). ${ }^{50} \mathrm{O}$ FBMN requer experimentos quantitativos (quantidade relativa) e uma etapa de préprocessamento de dados espectrais de modo que espectros de massas sejam convertidos a um feature. Nessa abordagem é necessário que as análises experimentais sejam realizadas por técnicas acopladas, como por exemplo, CLAE-EM e EM ${ }^{\mathrm{n}}$, uma vez que o tempo de retenção será associado aos espectros (feature é um conjunto de características que alia diferentes fontes de dados, por exemplo, sinais cromatográficos e espectrometria de massas).

Existem diversas ferramentas que realizam os pré-processamentos de dados espectrais como MZmine, ${ }^{51,52}$ OpenMS, ${ }^{53} \mathrm{MS}$-DIAL, ${ }^{54}$ MetaboScape, XCMS, ${ }^{55}$ Progenesis QI ou mzTab-M ${ }^{56}$ entre outras. ${ }^{46}$ Contudo, MZmine ${ }^{57}$ é a mais divulgada e utilizada pela comunidade de espectrometria de massas. Em geral, o pré-processamento necessário para a geração de features passa por nove etapas antes de ser carregada no FBMN.$^{50} \mathrm{O}$ Esquema 1 mostra, em detalhes, as etapas e os parâmetros (sugeridos) pelo fórum da comunidade de redes moleculares, para o pré-processamento de dados de $\mathrm{EM}^{\mathrm{n}}$ no MZmine. 


\begin{tabular}{|c|}
\hline$\frac{1 \text { - Importação dos Dados Brutos }}{\text { Raw data methods } \rightarrow \text { Raw data import }}$ \\
\hline 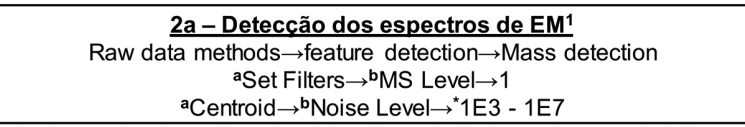 \\
\hline 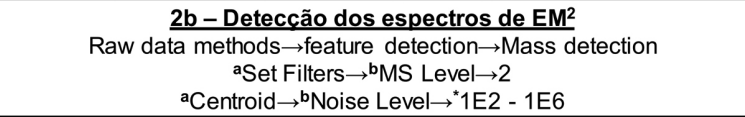 \\
\hline 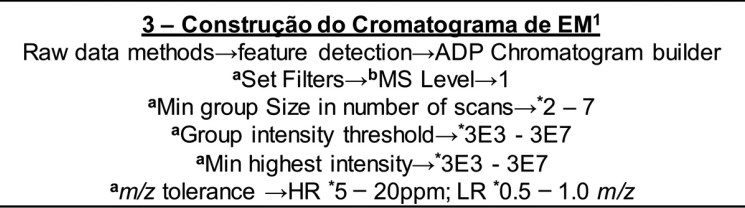 \\
\hline 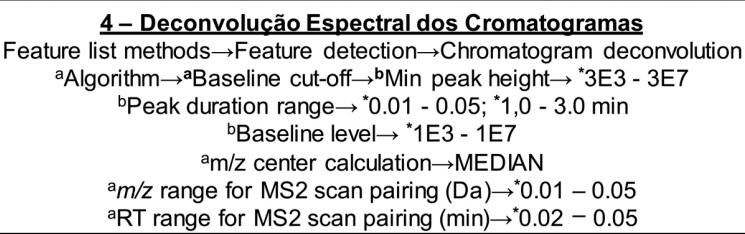 \\
\hline 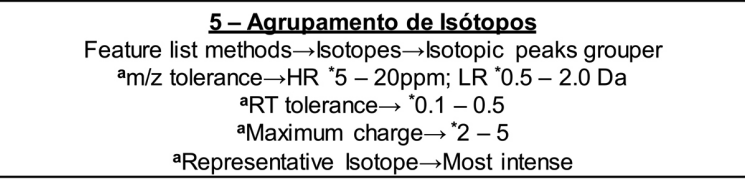 \\
\hline $\begin{array}{c}\underline{6-\text { Alinhamento }} \\
\text { aFeature list methods } \rightarrow \text { Alignment } \rightarrow \text { Join aligner } \\
\text { a } m / z \text { tolerance } \rightarrow H R{ }^{*} 5-20 p p m ; L R{ }^{*} 0.5-1.0 \mathrm{~m} / \mathrm{z} \\
\text { aWeight for } \mathrm{m} / \mathrm{z} \rightarrow{ }^{*} 50-75 \% \\
\text { aRetention time tolerance } \rightarrow 0.01-0.1 \mathrm{~min} \\
\text { aWeight for } \mathrm{RT} \rightarrow{ }^{*} 25-50 \%\end{array}$ \\
\hline $\begin{array}{l}\text { 7-Filtro (opcional) } \\
\text { Feature list methods } \rightarrow \text { Filtering } \rightarrow \text { Feature list rows filter } \\
\text { aMinimum peaks in a row } \rightarrow{ }^{*} 1-3 \\
\text { aMinimum peaks in an isotope pattern } \rightarrow \text { * } 1-3 \\
\text { aKeep only peaks with MS2 scan (GNPS) } \rightarrow \text { Sim }\end{array}$ \\
\hline $\begin{array}{l}\text { 8- Reanálise dos dados (opcional) } \\
\text { Feature list methods } \rightarrow \text { Gap filling } \rightarrow \text { Peak finder } \\
\text { alntensity tolerance } \rightarrow{ }^{*} 10 \% \\
{ }^{\mathrm{a}} \mathrm{m} / \mathrm{z} \text { tolerance } \rightarrow \text { HR }{ }^{*} 5-20 \mathrm{ppm} ; \mathrm{LR}{ }^{*} 0.5-1.0 \mathrm{~m} / \mathrm{z} \\
\text { aRetention time tolerance } \rightarrow{ }^{*} 0.01-0.1 \mathrm{~min}\end{array}$ \\
\hline $\begin{array}{c}\text { Feature list methods } \rightarrow \text { Export/lmport } \rightarrow \text { Export/Submit to GNPS-FBMN } \\
\text { aFilters rows } \rightarrow \text { Only with MS2 }\end{array}$ \\
\hline
\end{tabular}

Esquema 1. Pré-processamento de dados realizados no pacote Mzmine 2 para a geração de redes moleculares $(F B M N)$. ${ }^{a}$ representa a janela principal $e^{b}$ secundária. *os parâmetros aqui sugeridos não são absolutos, dependem do tipo de amostra, equipamentos e métodos; HR - alta resolução; LR - baixa resolução; " deve selecionar um arquivo no formato desejado

A primeira etapa consiste na importação dos dados brutos de CLAE-EM e $\mathrm{EM}^{\mathrm{n}}$ (ou técnicas cromatográficas similares como CLUE-EM e EM ${ }^{\mathrm{n}}$ ) e sua conversão em formato $m z X M L$; o segundo estágio corresponde a detecção e filtragem de íons presentes nos dados brutos. Nesse processamento define-se os limites de intensidade dos íons em $\mathrm{EM}^{1}$ e $\mathrm{EM}^{\mathrm{n}}$ visando excluir a maior quantidade possível de sinais de ruído. ${ }^{5,51} \mathrm{Na}$ sequência, são reconstruídos os cromatogramas de íons extraídos a partir de dados adquiridos, isto é, os íons extraídos anteriormente são rearranjados sequencialmente dando origem a um novo cromatograma. ${ }^{58} \mathrm{O}$ quarto estágio compreende-se a deconvolução dos cromatogramas. Esse processamento é importante para reconhecer e separar os isóbaros que possuem tempos de retenção diferentes e também promover a associação dos íons precursores com seus respectivos íons produto. ${ }^{51}$ Agrupar os íons que participam de um mesmo perfil isotópico em um único feature é a etapa seguinte, isótopos podem ser interpretados como substâncias diferentes, por isso é necessário reconhecê-los e agrupá-los sob um único feature. No sexto estágio ocorre o alinhamento dos features de cada amostra em uma única lista, nesta etapa é possível reconhecer as amostras que apresentam os mesmos features..$^{51} \mathrm{O}$ sétimo consiste em executar uma filtração na lista de dados que visa excluir os features provenientes de ruídos e interferentes. O oitavo estágio consiste em uma reanálise dos dados (Gap Filling), e tem o propósito de reestabelecer features de interesse que foram excluídos ou não selecionados por conta dos valores dos parâmetros utilizados. Entretanto, deve-se ter atenção com os parâmetros aplicados para não recuperar sinais que não são verdadeiros. ${ }^{50,51}$ Por fim os dados são exportados em dois arquivos, um arquivo no formato $c s v$, contendo as intensidades dos espectros de $\mathrm{EM}^{1}$ (com seus respectivos números de scans, possibilitando gerar maior interação e adições de novas informações aos features), e um no formato $m g f$, contendo todas as informações dos espectros de $\mathrm{EM}^{\mathrm{n}}$. Uma vez finalizado, esses arquivos podem ser submetidos ao fluxo de trabalho do FBMN. . $^{5,51,57,58}$

É possível ainda incluir uma tabela de metadados ${ }^{59}$ tanto no modo CMN quanto no FBMN.

\section{REDES MOLECULARES - ANÁLISE POR CG-IE-EM}

Redes moleculares a partir de dados de CG-IE-EM é uma opção recente na plataforma GNPS. Como os dados de massas oriundos de experimentos em IE não apresentam pré-seleção de íons precursores (formato de aquisição denominado de DIA) certas considerações são necessárias. Diversos conjuntos de dados podem ser combinados, entretanto é exigido um mesmo protocolo de análise, como o programa de temperatura, coluna, modo de injeção e energia de ionização. . $^{3,10,60}$

A plataforma do GNPS disponibiliza duas sessões para iniciar o trabalho de análise de dados CG-IE-EM. Na primeira é oferecido ao usuário a possibilidade de realização de um pré-processamento utilizando o MS-Hub, que além da deconvolução dos cromatogramas, típico de experimentos realizados em modo DDA, promove uma deconvolução espectral por meio de uma transformação rápida de Fourier, multiplicação e transformação inversa de Fourier para cada íon, seguida por uma fatoração de matriz não supervisionada. ${ }^{60}$ Todo processo é realizado de forma automática e dispensando qualquer expertise em estatística e/ou bioinformática. Entretanto para garantir a eficiência do processo, é crucial que no mínimo 10 amostras sejam utilizadas. A falta de informação pode levar a uma deconvolução instável, gerando espectros de baixa qualidade. ${ }^{60} \mathrm{E}$ possível utilizar outras opções de deconvolução como MZMine2/

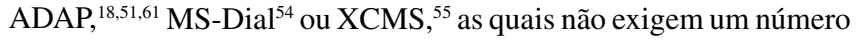
mínimo de amostras.

Transcorrida a etapa de pré-processamento, é gerado um arquivo $m g f$ contendo os espectros alinhados, deconvoluídos e com os tempos de retenção, bem como uma tabela contendo as áreas de pico detectados. Utilizando o MS-Hub, o arquivo é automaticamente introduzido no fluxo de trabalho do GNPS para comparação com a biblioteca espectral e, para construção da rede molecular, não exigindo etapas de download e upload no sistema. O usuário deve somente selecionar o link: Search Spectral Library and Molecular Network, disponível após a deconvolução dos espectros. O usuário também pode realizar seu download e utilizá-lo para explorar os resultados empregando diversas outras ferramentas ou bibliotecas, ${ }^{60}$ por exemplo MetExpert, ${ }^{62}$ NIST $2017^{63}$ e Wiley.

Tendo o usuário optado por utilizar o MS-Hub, o fluxo de trabalho para busca nas bibliotecas e criação da rede molecular será exibido automaticamente exigindo a definição de tolerância de massa para agrupamento dos espectros dos íons produtos, o número mínimo de íons produto e o valor mínimo de cosseno. Opções avançadas 
estão disponíveis, como da inclusão dos valores de referências de hidrocarbonetos para cálculo do índice de retenção (Kovats) ${ }^{60}$

\section{COMO UTILIZAR A PLATAFORMA GNPS E A FERRAMENTA DE VISUALIZAÇÃO CYTOSCAPE}

Para acessar a documentação e as ferramentas do GNPS é necessário incialmente fazer um cadastro através do website https://gnps.ucsd.edu. Para a construção da rede molecular, as análises e processamentos devem ser realizados de acordo com as recomendações da plataforma. Os dados devem incialmente ser convertidos em formatos do tipo $m z X M L, m z M L$, ou $m g f$ - pacotes como MSConvert, do grupo de ferramentas ProteoWizard realiza tais conversões, além da funcionalidade de conversão online usando o recurso arrastar e soltar disponível na plataforma GNPS, Figura 3. Existem duas opções para o carregamento dos dados na plataforma (upload): se os dados não ultrapassarem $20 \mathrm{MB}$, o upload pode ser realizado diretamente pelo site utilizando a função Drag and Drop; para arquivos maiores que $20 \mathrm{MB}$, protocolos de transferência do tipo FTP (File Transfer Protocol) são recomendados. O pacote livre WinSCP é indicado pela plataforma, onde o usuário acessa o servidor PreoteoSAFe - hospeda o GNPS e o MassIVE - e transfere os dados para a plataforma (outras ferramentas podem ser utilizadas como CoreFTP e CoffeeCup Free FTP). ${ }^{3}$

Uma vez convertidos e carregados, segue-se para etapa de configuração de parâmetros para a criação da rede molecular (para mais informações segue o link da documentação: https://ccms-ucsd. github.io/GNPSDocumentation/). De acordo com a particularidade do estudo, como o instrumento, técnica e poder de resolução, serão necessárias alterações nos parâmetros. O GNPS oferece campos de instrução para cada um deles. . $^{3,10}$

Não é recomendado o processamento simultâneo de dados obtidos em diferentes modos de ionização, porém o recurso Merge Network Polarity possibilita mesclar duas redes moleculares criadas em modos diferentes de ionização. Esse recurso pode ser utilizado tanto em redes criadas no CMN quanto no FBMN.

Embora a plataforma GNPS forneça a possibilidade de avaliação por ferramentas web browser, o uso de pacotes standalone faz-se necessário em casos de avaliação de redes em modo off-line e para conjuntos amostrais que necessitem maior poder de processamento. O Cytoscape ${ }^{64}$ é um destes programas (recomendado) de aplicação livre, feito pela comunidade científica para a visualização de grandes conjuntos de dados no formato de redes. Nele é possível realizar uma navegação dinâmica de gráficos, bem como utilizar de ferramentas de edição de rótulos, cores e formatos (os nodos são frequentemente associados a massa do íon precursor). Os arcos da rede frequentemente correspondem diferença de massa entre dois íons precursores ou o grau de similaridade entre eles.

A opção por web browser possibilita a rápida filtragem de tabelas do tipo html com ligações dinâmicas a bancos de dados espectrais, visualização de famílias moleculares (componentes conectados à rede), visualização dos espectros de fragmentação, comparação de espectros, geração de histogramas, além do gerenciamento e compartilhamento simplificado pelos web links. A possibilidade de gerenciar as tarefas, repetir as análises (clonar os parâmetros) e compartilhar com colaboradores tem motivado a ampla adoção da ferramenta pela comunidade científica..$^{3,10}$

\section{BIBLIOTECAS ESPECTRAIS E AS REDES MOLECULARES}

Um dos grandes desafios no processo anotação/identificação em química de produtos naturais é a carência de banco de dados públicos de espectros de massas sequencial $\left(\mathrm{EM}^{\mathrm{n}}\right) .{ }^{65} \mathrm{Na}$ tentativa de contornar esse obstáculo, muitos laboratórios acabam criando suas próprias bibliotecas (in house), que em sua maioria não são compartilháveis e/ou são incompatíveis com outras bibliotecas. Nesse sentido, o GNPS tem se empenhado na construção de uma biblioteca de produtos naturais comunitária, em que cada pesquisador pode acessar os espectros de referência gratuitamente, mas também depositar os seus próprios em um formato padronizado. ${ }^{3,10,65}$ Além disso, o GNPS disponibiliza informações de importantes bibliotecas de compostos como LDB Lichen Database, MIADB Spectral Library, ${ }^{12,66,67}$ Sumner Spectral Library, ${ }^{68}$ CASMI Spectral Library, ${ }^{66,69}$ MassBank (Japan (http:// massbank.jp), ${ }^{70}$ EU Mass Bank (https://massbank.eu/MassBank/) ${ }^{71} \mathrm{e}$ North America Mass Bank (http://mona.fiehnlab.ucdavis.edu/ ).

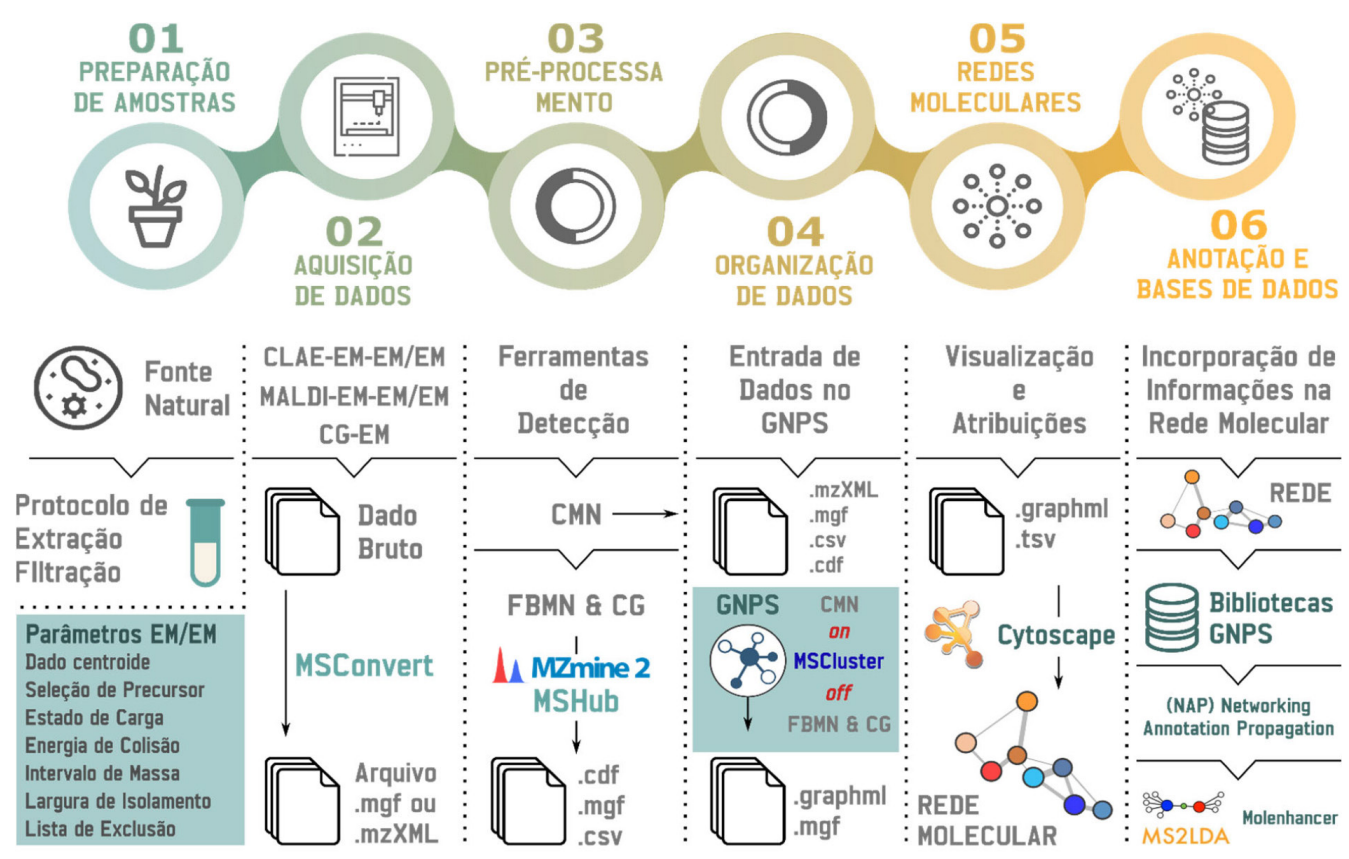

Figura 3. Ilustração do uso da ferramenta GNPS. São necessários 6 etapas entre a inserção e interpretação dos resultados. CMN - redes moleculares no modo clássico; FBMN-feature-based molecular networking; $C G$ - redes moleculares para dados de CG 
A forma mais comum de utilização da biblioteca é através da comparação automática dos espectros consenso das redes $\mathrm{CMN},{ }^{10}$ FBMN $^{50}$ ou CG-IE-EM,${ }^{60}$ contra os espectros da biblioteca espectral GNPS. ${ }^{3}$ Durante o fluxo de trabalho na plataforma o usuário pode selecionar as bibliotecas definindo o valor mínimo de cosseno (similaridade) e o número mínimo de íons fragmento comuns (entre o dado adquirido e o banco de espectros). ${ }^{10,50,60}$ Também é possível realizar a reanálise de um espectro específico empregando a ferramenta MASST, ela realiza uma nova busca na biblioteca espectral e permite alteração nos parâmetros de busca de forma individual. $^{72}$

Com Library Search é possível fazer buscas e comparações espectrais a partir de um único ou um conjunto espectros frente a biblioteca espectral. $\mathrm{O}$ usuário deve previamente converter seus dados para um formato compatível ( $m z X M L$ ou $m z M L)$ antes de carregá-los na plataforma. Em seguida, seleciona-se os arquivos, define-se a tolerância de massa em relação ao íon precursor e aos íons produto, o número mínimo de íons produto e o valor mínimo de cosseno. ${ }^{3}$ A principal diferença desse recurso daqueles que geram uma rede molecular é que não há alinhamento de espectros de consenso, ou seja, os espectros adquiridos de uma mesma substância serão considerados únicos.

Outra opção é o Molecular Explorer, ferramenta que permite o contato entre pesquisadores que possuem dados em comum. Utilizando do conceito de "dados vivos", o usuário pode buscar substâncias presentes em suas amostras, que foram relatadas para outros conjuntos de dados públicos. ${ }^{3,10}$ Por se tratar de um repositório público e colaborativo, também é possível acessar, explorar e realizar download de todos os espectros depositados na biblioteca espectral do GNPS. ${ }^{3}$

Além das buscas é possível contribuir com o banco comunitário de substância do GNPS. Todas as contribuições são revisadas e categorizadas baseadas na qualidade dos espectros e na confiabilidade das anotações. ${ }^{3,73}$ O GNPS classifica as contribuições utilizando três níveis de qualidade: (i) Gold (ouro) caracterizados por espectros de substâncias puras de origem sintética ou isolada de produtos naturais com caracterização estrutural completa, (ii) Silver (prata) os espectros são suportados por uma publicação e o (iii) Bronze (bronze), refere-se a caracterização incompleta ou não suportada por publicação. Os níveis ouro e prata, só podem ser atribuídos por membros autorizados da comunidade, mesmo se tratando de espectros de substâncias que preenchem os requisitos. A comunidade faz o depósito na categoria bronze e, após revisão, eles podem ser promovidos aos níveis superiores. Os formatos aceitos para depósito são $m z X M L, m z M L$ e $m g f^{3}$

\section{FERRAMENTAS INTEGRADAS AS REDES MOLECULARES}

As redes moleculares criaram uma forma estruturada de extrair e organizar dados de $\mathrm{EM}^{\mathrm{n}}$ contribuindo para a solução de muitos desafios em produtos naturais. ${ }^{74}$ No entanto, a anotação em larga escala de matrizes complexas continua sendo um obstáculo para a descoberta de novas entidades químicas bioativas. A taxa de anotação média, em estudos de redes moleculares pelo GNPS era de 1-2\% em 2012, data de sua primeira publicação, saltando para 5-6\% em nossos dias. ${ }^{10,74}$

Os metabólitos não anotados, também chamados de "matéria escura - alusão a matéria desconhecida no cosmo", representa o maior desafio para a química de produtos naturais, bioinformática e das ferramentas computacionais. ${ }^{18,75} \mathrm{Na}$ seção a seguir são mostradas ferramentas complementares as redes moleculares e ao processo de anotação: Network Annotation Promotion (NAP), ${ }^{74}$ MS2LDA, ${ }^{76,77}$ MolNetEnhancer ${ }^{78}$ e Qemistree. ${ }^{79}$

\section{Networking Annotation Propagation (NAP)}

O NAP é baseado no algoritmo de fragmentação do MetFrag ${ }^{80}$ e de ligação do MetFusion. ${ }^{81}$ Ele utiliza a rede molecular para melhorar a precisão das previsões in silico por meio da propagação de anotações estruturais ampliando as probabilidades de anotar corretamente as substâncias, mesmo sem uma correspondência com as bibliotecas espectrais. Isso é realizado através da criação de uma lista de candidatos que são reclassificados usando a topologia da rede molecular e similaridade estrutural, melhorando assim significativamente a classificação das anotações in silico. ${ }^{74}$ Apresentando uma interface de fácil aplicação, além do fato de estar integrado à plataforma GNPS, o pacote $\mathrm{NAP}^{74}$ pode ser utilizado logo após a construção das redes moelculares. ${ }^{10,50} \mathrm{O}$ NAP pode ser utilizado acessando o link https://gnps.ucsd.edu/ProteoSAFe/ static/gnps-theoretical.jsp, inserindo o JobID (identificador do processamento de uma rede, i.e., quando se cria uma rede no GNPS é gerado um ID) definindo alguns critérios para análise, como por exemplo, o valor mínimo de cosseno, modo de aquisição e a precisão de massa em ppm. Finalizado o processamento, os resultados podem ser explorados na própria plataforma ou exportado para o software Cytoscape ${ }^{64}$ NAP vem sendo empregado em diversos estudos na área de produto naturais, em que podemos destacar: avaliação da diversidade química de espécies do gênero Alnus,${ }^{82}$ no estudo multi-omics para avaliar o potencial de cianobactérias marinhas filamentosas tropicais ${ }^{83}$ e para anotação in silico dos marcadores de três espécies do gênero Zanthoxylum. ${ }^{84}$

\section{MS2LDA}

Inspirado na modelagem de texto por Latent Dirichlet Allocation (LDA), ${ }^{85}$ MS2LDA é uma ferramenta que decompõe dados de fragmentação molecular e identifica padrões de fragmentação de massas. Fragmentos co-ocorrentes e/ou perdas neutras que representam subestruturas moleculares relevantes (p. ex., hexose ou a perda de um grupo carboxila) potencialmente indicativos de famílias estruturais, denominados Mass2Motifs, são estruturalmente caracterizados e utilizados para anotar moléculas para as quais não existem espectros de referência. ${ }^{76,77}$

A ferramenta MS2LDA está integrada à plataforma GNPS e pode ser selecionada através da opção Analyze with MS2LDA na página resultados, disponível no final do processamento da rede molecular. Uma vez que o processamento seja finalizado é possível explorar os resultados tanto na plataforma ou exportando para o software Cytoscape $^{64}$ ou na página do MS2LDA. ${ }^{76,77}$

Assim como as bibliotecas comunitárias nas quais o usuário pode depositar/anotar uma substância com base no seu espectro, o MS2LDA oferece um serviço de anotação de motifs. Essa funcionalidade é uma das grandes inovações da aplicação, uma vez que facilita e acelera a anotação de substâncias a partir dos motifs identificados (fragmentos).

Recentemente um trabalho utilizando redes moleculares e MS2LDA foi capaz de indicar a presença de novos ésteres de diterpenos em espécies do gênero Euphorbia com atividade inibitória contra o vírus Chikungunya. ${ }^{48}$

\section{MolNetEnhancer}

A fim de integrar algumas ferramentas complementares disponíveis no GNPS às redes moleculares foi desenvolvido a ferramenta MolNetEnhancer. ${ }^{78}$ Esta aplicação proporciona a combinação de uma rede molecular com ferramentas in silico como o $\mathrm{NAP}^{74}$ e o MS2LDA ${ }^{76}$ e também automatiza a 
classificação química da ferramenta ClassyFire. ${ }^{86}$ Isso permite a visualização de classes e subclasses químicas presente nos clusters, incrementando as informações estruturais. ${ }^{78} \mathrm{Na}$ seção das ferramentas avançadas, subseção ferramentas experimentais, pode- se utilizar o MolNetEnhancer inserindo os JobsIDs das redes moleculares, NAP e MS2LDA. Ao término do processamento é possível exportá-los para o Cytoscape. ${ }^{64}$

Uma das primeiras aplicações do MolNetEnhancer foi no estudo metabolômico de superfoods. Os autores observaram que os superfoods tiveram uma maior taxa de anotação de compostos quando comparados aos não superfoods. ${ }^{87}$

\section{Qemistree}

O Qemistree ${ }^{79}$ é uma ferramenta que combina as redes moleculares do tipo $\mathrm{FBMN}^{50}$ com as ferramentas in silico, SIRIUS $^{88}$ e CSI:FingerID, ${ }^{89}$ e constrói uma árvore química com base nas impressões digitais moleculares. Isso permite avaliações ecológicas de dados químicos em relação as sequências de DNA geradas para árvores taxonômicas (disponíveis) de espécies, gêneros e famílias. ${ }^{79}$ Para simplificar sua aplicação, o fluxo de trabalho do Qemistree pode ser acessado diretamente da página de resultados da rede molecular processada através da opção: Visualize with Qemistree. Automaticamente o fluxo de trabalho será carregado requisitando apenas um arquivo $m g f$, contendo as informações dos espectros gerados para a plataforma do SIRIUS através do pacote MZmine 2 (Esquema 1), seleção do tipo de analisador e modo de ionização dos experimentos. Uma vez que o processamento seja finalizado, as árvores químicas são visualizadas e exploradas através das ferramentas iTOL $^{90}$ e QIIME2.91

\section{APLICAÇÃO DE REDES MOLECULARES A QUÍMICA DE PRODUTOS NATURAIS}

Um levantamento realizado na Web of Science (ISI), empregando como palavras-chave, "molecular networking" e "natural products" apontou que 711 trabalhos foram publicados em periódicos internacionais especializados entre 2013 e 2021 ( 01 de janeiro de 2013 a 20 março de 2021), como mostrado na Figura 4. Uma inspeção detalhada dos resultados confirmou a aplicação de redes moleculares em estudos de desreplicação, priorização de extratos e frações, exploração de interações ecológicas, caracterização de genes e vias biossintéticas. A versatilidade das redes moleculares, frequentemente

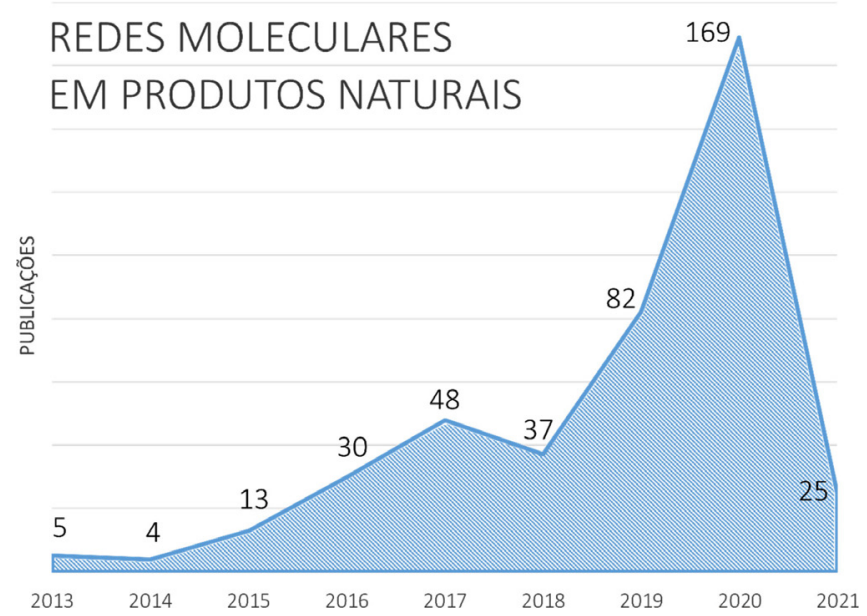

Figura 4. Levantamento de artigos científicos publicados entre 2013 e 2021 pela Web of Science utilizando como palavras-chave: Molecular Networking e Natural Products associada a metabolômica e aos produtos naturais, reflete o crescente número e qualidade das publicações.

Contudo, o surgimento de novas ferramentas exige um período de adaptação. Até o momento, a capacidade média de anotação do GNPS não ultrapassou $20 \%$ dos íons totais para um estudo. Por outro lado, a anotação com base em reações de fragmentação de um íon,,$^{42}$ presente em um nodo, pode levar a novas estruturas ou permitir o isolamento guiado para a completa caracterização de novos produtos naturais. ${ }^{47}$

\section{Estudos bioguiados e priorização de frações e extratos para anotação e isolamento de novos quimiotipos bioativos}

Estudos de desreplicação e descoberta de novos agentes bioativos a partir de matrizes biológicas complexas (produtos naturais) são os tipos de estudos mais aplicados em redes moleculares. Naman e colaboradores $^{92}$ analisaram 10 cianobactérias do gênero Symploca sp. na busca por substâncias citotóxicas. As frações analisadas pelas redes moleculares e a biblioteca do GNPS levou a identificação de um peptídeo inédito com ação citotóxica.

Palicourea sessilis (Família Rubiaceae) foi analisada por redes moleculares, com a utilização do Dictionary of Natural Products combinado a ferramenta de fragmentação in silico DNP-ISDB. Os autores identificaram e, posteriormente, confirmaram por isolamento, oito alcaloides monoterpenicos, sendo três inéditos com moderada atividade anticolinesterásica. ${ }^{93}$

Olivon e colaboradores ${ }^{94}$ utilizaram as redes moleculares em combinação com dados de bioatividade contra o vírus Chikungunya e sua relação de sinalização Wnt (regulador de eventos embrionários) sob um conjunto taxonômico de 107 espécies de Euphorbiaceae (Nova Caledonian). 292 extratos (ativos e inativos) foram gerados, entre extratos de folhas, cascas, galhos, plantas e frutos inteiros. O estudo levou a priorização e posteriormente ao isolamento e elucidação de quatro ésteres de 12-desoxiforbol (dois inéditos) com atividade contra o vírus Chikungunya.

O estudo bioguiado com Angelica keiskei (Apiaceae) e redes moleculares levou à descoberta de chalconas com propriedades antimicrobianas contra Staphylococcus aureus. Os resultados da rede foram analisados por ferramentas quimiométricas, indicando o agrupamento das chalconas como responsável pela ação antimicrobiana dos extratos e frações. Foram isolados análogos como o xanthoangelol K, cuja atividade antimicrobiana foi pela primeira vez reportada. ${ }^{95}$

Hou e colaboradores, ${ }^{96}$ utilizando dados de CLAE-EM e $\mathrm{EM}^{\mathrm{n}}$, redes moleculares e dados de $\mathrm{RMN}$ de ${ }^{1} \mathrm{H}$, identificaram ciclohexadepsipeptídeos bioativos (crysogeamindas A -G) de Penicillium chrysogenun.

Redes moleculares combinadas a bancos de dados in house foram utilizadas para estudos de cascas de Geissospermum laeve (Família Apocynaceae). Três novos alcaloides indólicos monoterpenicos foram encontrados. O estudo ainda realizou ensaios antiparasitários e citotóxicos para as substâncias isoladas. ${ }^{36}$

A estratégia metabolômica baseada em espectrometria de massas e redes moleculares também foi recentemente utilizada para caracterizar compostos com atividade larvicida contra Aedes aegypti. Utilizando o MetaboAnalyst, redes moleculares e ensaios biológicos para os extratos da espécie Annona crassiflora foram isolados e caracterizados diversas acetogenias ativas contra as larvas do mosquito Aedes aegypti (principal responsável pela transmissão de dengue e outras arboviroses no Brasil).$^{38}$

Redes moleculares têm sido aplicadas também para a caracterização de compostos anticancer ${ }^{97} \mathrm{e}$ antibiofilme ${ }^{98}$ de organismos marinhos. Contudo, o grande potencial dessa estratégia pode ser verificado pelo trabalho de Floros e colaboradores ${ }^{99}$ analisando cerca 
de 1000 microrganismos marinhos, dos quais mais de $70 \%$ não haviam sido taxonomicamente caracterizados. Os autores avaliaram o espaço químico de produtos naturais marinhos priorizando extratos na ausência de informações genômicas ou de atividade biológica. Agrupamentos específicos para algumas cepas levaram ao isolamento e elucidação de duas substâncias novas, os ácidos marídricos A e B..$^{99}$

Uma derivação mais recente das redes moleculares, denominada bioactive molecular network, foi desenvolvida por Nothias e colaboradores para acelerar a priorização e isolamento de substâncias em estudos bioguiados. ${ }^{100} \mathrm{O}$ valor de bioatividade dos extratos (independente do teste de bioatividade) é associado as quantificações dos íons detectados nos experimentos de EM $\left(\mathrm{EM}^{1}\right)$ nas redes moleculares. Após identificar as correlações significativas, os dados são visualizados através da rede, indicando os nodos bioativos, facilitando assim seu processo de desreplicação. Contudo, é importante ressaltar que a intensidade dos íons detectados pode não refletir a realidade da concentração, uma vez que essa intensidade está diretamente relacionada a afinidade protônica ou a capacidade de coordenação. ${ }^{101}$

\section{Interações químicas, ecológicas e variação metabólica}

Existem diversos estudos em redes moleculares relacionados à ecologia química, incluindo relações de comensalismo, mutualismo e simbiose, para as interações positivas, como também relações de predação, parasitismo e antibiose. ${ }^{8,102}$ Entre as diferentes ferramentas ômicas utilizadas para compreensão destes fenômenos, ${ }^{103}$ as redes moleculares ganharam destaque para eventos de sinalização ou defesa química.

Caraballo-Rodríguez, ${ }^{104}$ observou a expressão diferenciada de metabólitos produzidos por endófitos (actinobactérias e fungos) de Lychnophora ericoides (Asteraceae) sob diferentes formas de tratamento. Culturas isoladas e co-cultivos mostraram que actinobactérias afetaram negativamente o crescimento e desenvolvimento dos fungos. A rede molecular indicou algumas classes e análogos responsáveis pelo processo de inibição dos fungos.

Vallet et al. ${ }^{105}$ notaram biotransformações de surfactinas em experimentos de co-cultivo entre Bacillus subtilis e o fungo Paraconiothyrium variabile (endófíto de Cephalotaxus harringtonia - Taxaceae). Análogos presentes nos agrupamentos das surfactinas apontaram uma diferença de massa de $18 \mathrm{Da}$, indicando uma perda de água entre os nodos do cluster. Esse estudo sugere uma interação interespecífica, onde a perda de água das surfactinas produzidas pela bactéria aumenta toxicidade e resistência contra os fungos.

Em um estudo brasileiro com espécies de Palitoa, que habitam o mesmo ambiente dos corais, foi observado um efeito ambiental na química das espécies estudadas. ${ }^{106}$

Redes moleculares foram usadas para avaliação química de recifes de corais distribuídos em diferentes locais do planeta. Os autores apontaram diferenças de massas para grupos putativos como $\mathrm{H}_{2}, \mathrm{CH}_{2}, \mathrm{COCH}_{2}$, dependendo da origem do táxon. ${ }^{107}$ Dessa forma, a rede molecular foi capaz de ajudar a anotar de modo simplificado a variação metabólica que ocorre nesses holobiontes, mostrando como o ambiente afeta diretamente a produção metabólica.

Um estudo metabolômico utilizando redes moleculares, possibilitou a identificação dos metabólitos responsáveis pela resistência em sojas contaminadas pelo fungo causador da ferrugem asiática. $\mathrm{O}$ estudo mostra o potencial da estratégia para o controle de pragas. ${ }^{108}$ Finalmente, um estudo utilizando de redes moleculares para caracterização de bileverdina, serviu de partida para a identificação de um complexo metabólito-proteína de cor azul, que sob a pele amarela das rãs, confere um tom esverdeado utilizado em processos de camuflagem em folhas. ${ }^{109}$

\section{Genômica funcional, estudos de biossíntese via redes moleculares}

A associação da rede molecular com estudos genéticos e biossíntese também tem ganhado destaque na área de produtos naturais. A análise de três espécies de cianobactérias marinhas Moorea producens 3L, Moorea producens JHB, e Moorea bouillonii PNG levou à descoberta de três novas substâncias pertencentes a uma nova classe, chamadas de columbamidas A, B e C. As cianobactérias foram submetidas às análises genômicas comparando as informações obtidas com as análises metabolômica através das redes moleculares. ${ }^{110}$

Uma abordagem similar foi descrita por Maansson et al., ${ }^{111}$ a partir de estudos genômicos combinado às redes moleculares para análise de metabólitos bioativos de treze bactérias marinhas Gram-negativas. As análises de pan-plots e core-plots apontaram que apenas $2 \%$ das características foram compartilhadas entre todas as linhagens, enquanto $30 \%$ foram específicas a uma única linhagem. A rede molecular apontou um agrupamento característico das substâncias conhecidas e guiou a descoberta de dois novos análogos de tiomarinol.

Parkinson e colaboradores ${ }^{112}$ também utilizaram a metabolômica, redes moleculares e genômica para a descoberta de novos produtos naturais. Dois peptídeos não ribossomais produzidos por espécies da actinobactéria do gênero Streptomyces e possíveis análogos estavam agrupados em um mesmo cluster, os quais foram posteriormente caracterizados pelas vias biossintéticas.

O fungo Aspergillus nidulans foi estudado utilizando aminoácidos marcados com isótopos estáveis (SILAAs) e redes moleculares para caracterização de suas vias biossintéticas. O cultivo possibilitou a determinação da via da nudalina $\mathrm{A}$ e seus análogos, bem como a anotação de fungisporina, ainda não reportada para a espécie. ${ }^{113}$

Outro exemplo importante, nesse contexto, ocorreu pela aplicação de proteômica top-down, redes moleculares e marcação isotópica para a caracterização da via biossíntetica de um peptídeo não-ribossomal raro. ${ }^{114}$

A Tabela 1 mostra outros estudos não relatados até aqui empregando redes moleculares em conjunto com as ferramentas complementares em produtos naturais.

\section{CONCLUSÕES}

A abordagem por redes moleculares está entre os grandes avanços das ferramentas computacionais aplicada a química de produtos naturais do século XXI. A visualização de forma estruturada e intuitiva de grandes conjuntos amostrais facilita a compreensão de eventos multifatoriais encontrados em ecologia química, biossíntese, quimiotaxonomia, e principalmente na desreplicação e na descoberta de entidades químicas bioativas. Contudo, como em qualquer abordagem, seu uso indiscriminado e não supervisionado pode levar a erros de anotação. Portanto, recomenda-se uma curagem dos compostos de interesse usando uma abordagem de double-check.

$\mathrm{O}$ aspecto comunitário das redes moleculares, como a troca de informações entre autores e dados experimentais, é outra importante conquista para o campo dos produtos naturais. A expansão das bases de dados experimentais a partir de esforços individuais - geração de coleções espectrais de alta qualidade - podem ser agrupadas e disponibilizadas, aumentando o grau de confiabilidade das anotações em matrizes biológicas complexas. Os avanços nos protocolos e métodos de detecção ortogonais (tais como RMN, DAD, ELSD e CAD) são fatores necessários para uma completa caracterização dos compostos presentes nas redes. Nesse sentido, é importante ressaltar que a principal funcionalidade das redes moleculares é organização e visualização intuitiva de grandes conjuntos de dados, 
Tabela 1. Trabalhos utilizando redes moleculares para estudos em produtos naturais

\begin{tabular}{|c|c|c|c|c|}
\hline Fonte Natural & Tipo de Estudo & Relevância & Ferramentas Computacionais & Ref. \\
\hline $\begin{array}{l}\text { Abelhas e colônias } \\
\text { microbianas }\end{array}$ & Ecologia Química & $\begin{array}{l}\text { A importância dos flavonoides } \\
\text { para sobrevivência de abelhas }\end{array}$ & CMN/PCA/OPLS-DA & Silva-Junior ${ }^{115}$ \\
\hline Actinobactérias & $\begin{array}{c}\text { Ecologia Química e } \\
\text { Biotransformação }\end{array}$ & $\begin{array}{c}\text { Halogenação natural de } \\
\text { flavonoides por actinobactérias }\end{array}$ & $\mathrm{CMN}$ & Lee $^{116}$ \\
\hline Bactérias Marinhas & Bioatividade & $\begin{array}{c}\text { Estudo da variação dos } \\
\text { parâmetros de crescimento } \\
\text { para a produção de metabólitos } \\
\text { bioativos }\end{array}$ & FBMN & Conde-Martínez $z^{117}$ \\
\hline Barata do Mar & Bioatividade & $\begin{array}{l}\text { Analgésico e anti-inflamatório } \\
\text { de derivados do ácido Z-9- } \\
\text { hexadecenóico }\end{array}$ & CG-EM & Yue $^{118}$ \\
\hline Corais & Ecologia e Ambiental & $\begin{array}{c}\text { Diferenciação do metabolismo } \\
\text { de dois zoantários coletados ao } \\
\text { longo da costa brasileira }\end{array}$ & $\mathrm{CMN}$ & Costa-Lotufo ${ }^{106}$ \\
\hline Esponja marinha & Caracterização & Quinoides azuis zwitteriônicos & $\mathrm{CMN}$ & Bonneau $^{119}$ \\
\hline Fungo fitopatogênico & Biossíntese & $\begin{array}{c}\text { Elucidação da rota biosintética } \\
\text { de dibenzoespirocetais }\end{array}$ & $\mathrm{CMN}$ & $\mathrm{Zhu}^{120}$ \\
\hline Fungos & Biotransformação & $\begin{array}{l}\text { Oxidações do anéis } \mathrm{B} \text { e } \mathrm{C} \text { de } \\
\text { galatos de epigalocatequinas }\end{array}$ & FBMN & $\mathrm{Xie}^{45}$ \\
\hline Fungos de formigas & Ecologia & $\begin{array}{c}\text { Produção metabólica da } \\
\text { interação de fungos e bactérias }\end{array}$ & $\mathrm{CMN}$ & Boya $^{121}$ \\
\hline Plantas & Bioatividade & $\begin{array}{l}\text { alcaloides 3-aminospirostanos } \\
\text { anotados por redes moleculares }\end{array}$ & $\mathrm{CMN}$ & Gazolla $^{47}$ \\
\hline Plantas & Desreplicação e bioatividade & $\begin{array}{c}\text { Elucidação e priorização de } \\
\text { alcaloides quinolínicos com } \\
\text { atividade tripanocida }\end{array}$ & $\mathrm{CMN}$ & Gaudry ${ }^{122}$ \\
\hline Plantas & Anotação & $\begin{array}{c}\text { Estudo de fragmentação de } \\
\text { flavonoides glicosilados }\end{array}$ & $\mathrm{CMN}$ & Pilon $^{9}$ \\
\hline Plantas & Biossíntese & $\begin{array}{c}\text { Elucidação da rota metabólica } \\
\text { de alcaloides eritrínicos }\end{array}$ & FBMN & Chacon $^{123}$ \\
\hline Sapos & Ecologia Química & $\begin{array}{l}\text { Primero relato de fluorescência } \\
\text { em Sapos }\end{array}$ & $\mathrm{CMN}$ & Taboada $^{49}$ \\
\hline
\end{tabular}

e que o processo de anotação e principalmente de identificação de compostos é dependente de estratégias complementares, que podem ser através metodologias de desreplicação ou por meio de isolamento e caracterização utilizando de técnicas clássicas em PN.

Como perspectiva, a abordagem pode incorporar outras funcionalidades como dados quimiométricos, genômicos e/ou farmacológicos de modo a gerar uma plataforma multifuncional para a pesquisa em produtos naturais.

\section{AGRADECIMENTOS}

Os autores agradecem à Coordenação de Aperfeiçoamento de Pessoal de Nível Superior (CAPES), à Fundação de Amparo à Pesquisa do Estado de São Paulo (FAPESP - ACP [\#2019/15889-0 e 2016/13286-8] e à RRS [\#17/18922-2 e \#19/05026-4]), à FINATEC pela bolsa concedida a ACP - \#807/2020), ao Conselho Nacional de Desenvolvimento Científico e Tecnológico pela bolsa concedida a AFM [153221/2018-6] e ao CNPq pelo projeto concedido a JGA (\#436333/2018-0). Agradecemos também ao especial apoio da FAPESP ao projeto temático sob coordenação de NPL.

\section{REFERÊNCIAS}

1. Gaudêncio, S. P.; Pereira, F.; Nat. Prod. Rep. 2015, 32, 779.

2. Valli, M.; Russo, H. M.; Pilon, A. C.; Pinto, M. E. F.; Dias, N. B.; Freire, R. T.; Castro-Gamboa, I.; Bolzani, V. D. S.; Phys. Sci. Rev. 2019, 4.

3. Wang, M.; Carver, J. J.; Phelan, V. V.; Sanchez, L. M.; Garg, N.; Peng, Y.; Nguyen, D. D.; Watrous, J.; Kapono, C. A.; Luzzatto-Knaan, T.; Porto, C.; Bouslimani, A.; Melnik, A. V.; Meehan, M. J.; Liu, W. T.; Crüsemann, M.; Boudreau, P. D.; Esquenazi, E.; Sandoval-Calderón, M.; Kersten,
R. D.; Pace, L. A.; Quinn, R. A.; Duncan, K. R.; Hsu, C. C.; Floros, D. J.; Gavilan, R. G.; Kleigrewe, K.; Northen, T.; Dutton, R. J.; Parrot, D.; Carlson, E. E.; Aigle, B.; Michelsen, C. F.; Jelsbak, L.; Sohlenkamp, C.; Pevzner, P.; Edlund, A.; McLean, J.; Piel, J.; Murphy, B. T.; Gerwick, L.; Liaw, C. C.; Yang, Y. L.; Humpf, H. U.; Maansson, M.; Keyzers, R. A.; Sims, A. C.; Johnson, A. R.; Sidebottom, A. M.; Sedio, B. E.; Klitgaard, A.; Larson, C. B.; Boya, C. A. P.; Torres-Mendoza, D.; Gonzalez, D. J.; Silva, D. B.; Marques, L. M.; Demarque, D. P.; Pociute, E.; O’Neill, E. C.; Briand, E.; Helfrich, E. J. N.; Granatosky, E. A.; Glukhov, E.; Ryffel, F.; Houson, H.; Mohimani, H.; Kharbush, J. J.; Zeng, Y.; Vorholt, J. A.; Kurita, K. L.; Charusanti, P.; McPhail, K. L.; Nielsen, K. F.; Vuong, L.; Elfeki, M.; Traxler, M. F.; Engene, N.; Koyama, N.; Vining, O. B.; Baric, R.; Silva, R. R.; Mascuch, S. J.; Tomasi, S.; Jenkins, S.; Macherla, V.; Hoffman, T.; Agarwal, V.; Williams, P. G.; Dai, J.; Neupane, R.; Gurr, J.; Rodríguez, A. M. C.; Lamsa, A.; Zhang, C.; Dorrestein, K.; Duggan, B. M.; Almaliti, J.; Allard, P. M.; Phapale, P.; Nothias, L. F.; Alexandrov, T.; Litaudon, M.; Wolfender, J. L.; Kyle, J. E.; Metz, T. O.; Peryea, T.; Nguyen, D. T.; VanLeer, D.; Shinn, P.; Jadhav, A.; Müller, R.; Waters, K. M.; Shi, W.; Liu, X.; Zhang, L.; Knight, R.; Jensen, P. R.; Palsson, B.; Pogliano, K.; Linington, R. G.; Gutiérrez, M.; Lopes, N. P.; Gerwick, W. H.; Moore, B. S.; Dorrestein, P. C.; Bandeira, N.; Nat. Biotechnol. 2016, 34,828 .

4. Allard, P. M.; Genta-Jouve, G.; Wolfender, J. L.; Curr. Opin. Chem. Biol. 2017, 36, 40 .

5. Pilon, A. C.; Selegato, D. M.; Fernandes, R. P.; Bueno, P. C. P.; Pinho, D. R.; Neto, F. C.; Freire, R. T.; Castro-Gamboa, I.; Bolzani, V. S.; Lopes, N. P.; Quim. Nova 2020, 43, 329.

6. Bueno, P. C. P.; Lopes, N. P.; ACS Omega 2020, 5, 1752.

7. Ernst, M.; Silva, D. B.; Silva, R. R.; Vêncio, R. Z. N.; Lopes, N. P.; Nat. Prod. Rep. 2014, 31, 784. 
8. Fox Ramos, A. E.; Evanno, L.; Poupon, E.; Champy, P.; Beniddir, M. A.; Nat. Prod. Rep. 2019, 36, 960.

9. Pilon, A. C.; Gu, H.; Raftery, D.; Bolzani, V. D. S.; Lopes, N. P.; CastroGamboa, I.; Carnevale Neto, F.; Anal. Chem. 2019, 91.

10. Aron, A. T.; Gentry, E. C.; McPhail, K. L.; Nothias, L. F.; NothiasEsposito, M.; Bouslimani, A.; Petras, D.; Gauglitz, J. M.; Sikora, N.; Vargas, F.; van der Hooft, J. J. J.; Ernst, M.; Kang, K. Bin; Aceves, C. M.; Caraballo-Rodríguez, A. M.; Koester, I.; Weldon, K. C.; Bertrand, S.; Roullier, C.; Sun, K.; Tehan, R. M.; Boya P, C. A.; Christian, M. H.; Gutiérrez, M.; Ulloa, A. M.; Tejeda Mora, J. A.; Mojica-Flores, R.; Lakey-Beitia, J.; Vásquez-Chaves, V.; Zhang, Y.; Calderón, A. I.; Tayler, N.; Keyzers, R. A.; Tugizimana, F.; Ndlovu, N.; Aksenov, A. A.; Jarmusch, A. K.; Schmid, R.; Truman, A. W.; Bandeira, N.; Wang, M.; Dorrestein, P. C.; Nat. Protoc. 2020, 15, 1954.

11. Yang, J. Y.; Sanchez, L. M.; Rath, C. M.; Liu, X.; Boudreau, P. D.; Bruns, N.; Glukhov, E.; Wodtke, A.; De Felicio, R.; Fenner, A.; Wong, W. R.; Linington, R. G.; Zhang, L.; Debonsi, H. M.; Gerwick, W. H.; Dorrestein, P. C.; J. Nat. Prod. 2013, 76, 1686.

12. Watrous, J.; Roach, P.; Alexandrov, T.; Heath, B. S.; Yang, J. Y.; Kersten, R. D.; Van Der Voort, M.; Pogliano, K.; Gross, H.; Raaijmakers, J. M.; Moore, B. S.; Laskin, J.; Bandeira, N.; Dorrestein, P. C.; Proc. Natl. Acad. Sci. U. S. A. 2012, 109, 1743.

13. Cortelo, P. C.; Demarque, D. P.; Dusi, R. G.; Albernaz, L. C.; BrazFilho, R.; Goncharova, E. I.; Bokesch, H. R.; Gustafson, K. R.; Beutler, J. A.; Espindola, L. S.; Cells 2021, 10, 691.

14. Mannochio-Russo, H.; Bueno, P. C. P.; Bauermeister, A.; de Almeida, R. F.; Dorrestein, P. C.; Cavalheiro, A. J.; Bolzani, V. S.; J. Nat. Prod. 2020, $83,3239$.

15. Silva, E.; Perez da Graça, J.; Porto, C.; Martin do Prado, R.; Nunes, E.; Corrêa Marcelino-Guimarães, F.; Conrado Meyer, M.; Jorge Pilau, E.; Metabolites 2021, 11, 179.

16. Tangerina, M. M. P.; Furtado, L. C.; Leite, V. M. B.; Bauermeister, A.; Velasco-Alzate, K.; Jimenez, P. C.; Garrido, L. M.; Padilla, G.; Lopes, N. P.; Costa-Lotufo, L. V.; Pena Ferreira, M. J.; PLoS One 2020, 15, e0244385.

17. Santos, C. L. G.; Angolini, C. F. F.; Neves, K. O. G.; Costa, E. V.; Souza, A. D. L.; Pinheiro, M. L. B.; Koolen, H. H. F.; Silva, F. M. A.; Rapid Commun. Mass Spectrom. 2020, 34.

18. Soares, V.; Taujale, R.; Garrett, R.; da Silva, A. J. R.; Borges, R. M.; Phytochem. Anal. 2019, 30, 132.

19. Calheiros de Carvalho, A.; De Camillis Rodrigues, L.; Ribeiro, A. I.; Fernandes da Silva, M. F. das G.; Soman de Medeiros, L.; Moura Veiga, T. A.; Molecules 2019, 25, 153.

20. Paulo, B. S.; Sigrist, R.; Angolini, C. F. F.; De Oliveira, L. G.; ChemistrySelect 2019, 4, 7785.

21. Paz, W. H. P.; de Oliveira, R. N.; Heerdt, G.; Angolini, C. F. F.; S. de Medeiros, L.; Silva, V. R.; Santos, L. S.; Soares, M. B. P.; Bezerra, D. P.; Morgon, N. H.; Almeida, J. R. G. S.; da Silva, F. M. A.; Costa, E. V.; Koolen, H. H. F.; J. Nat. Prod. 2019, 82, 2220.

22. de Almeida, R. T. R.; do Prado, R. M.; Porto, C.; dos Santos, G. T.; Huws, S. A.; Pilau, E. J.; Sci. Rep. 2018, 8, 17971.

23. Bauermeister, A.; Velasco-Alzate, K.; Dias, T.; Macedo, H.; Ferreira, E. G.; Jimenez, P. C.; Lotufo, T. M. C.; Lopes, N. P.; Gaudêncio, S. P.; Costa-Lotufo, L. V.; Front. Microbiol. 2018, 9.

24. Philippus, A. C.; Zatelli, G. A.; Wanke, T.; Gabriela de A. Barros, M.; Kami, S. A.; Lhullier, C.; Armstrong, L.; Sandjo, L. P.; Falkenberg, M.; RSC Adv. 2018, 8, 29654.

25. da Silva, R. R.; Wang, M.; Nothias, L.-F.; van der Hooft, J. J. J.; Caraballo-Rodríguez, A. M.; Fox, E.; Balunas, M. J.; Klassen, J. L.; Lopes, N. P.; Dorrestein, P. C.; PLOS Comput. Biol. 2018, 14, e1006089.

26. Trivella, D. B. B.; de Felicio, R.; mSystems 2018, 3.

27. de Oliveira, G.; Carnevale Neto, F.; Demarque, D.; de Sousa PereiraJunior, J.; Sampaio Peixoto Filho, R.; de Melo, S.; da Silva Almeida, J.;
Lopes, J.; Lopes, N.; Planta Med. 2016, 83, 636.

28. Caraballo-Rodríguez, A. M.; Dorrestein, P. C.; Pupo, M. T.; Sci. Rep. 2017, 7, 5373

29. Kim, S.; Chen, J.; Cheng, T.; Gindulyte, A.; He, J.; He, S.; Li, Q.; Shoemaker, B. A.; Thiessen, P. A.; Yu, B.; Zaslavsky, L.; Zhang, J.; Bolton, E. E.; Nucleic Acids Res. 2021, 49, D1388.

30. Smith, C. A.; Maille, G. O.; Want, E. J.; Qin, C.; Trauger, S. A.; Brandon, T. R.; Custodio, D. E.; Abagyan, R.; Siuzdak, G.; Ther. Drug Monit. 2005, 27, 747.

31. Shen, V. K.; Siderius, D. W.; Krekelberg, W. P.; Hatch, H. W.; NIST Stand. Ref. Database 2017, 2014.

32. Kopka, J.; Schauer, N.; Krueger, S.; Birkemeyer, C.; Usadel, B.; Bergmüller, E.; Dörmann, P.; Weckwerth, W.; Gibon, Y.; Stitt, M.; Willmitzer, L.; Fernie, A. R.; Steinhauser, D.; Bioinformatics 2005, 21, 1635.

33. Schulte, C. F.; Tolmie, D. E.; Maziuk, D.; Nakatani, E.; Akutsu, H.; Yao, H.; others; Nucleic Acids Res. 2007, 36, D402.

34. Pilon, A. C.; Valli, M.; Dametto, A. C.; Pinto, M. E. F.; Freire, R. T.; Castro-Gamboa, I.; Andricopulo, A. D.; Bolzani, V. S.; Sci. Rep. 2017, 7.

35. Bandeira, N.; Tsur, D.; Frank, A.; Pevzner, P. A.; Proc. Natl. Acad. Sci. U. S. A. 2007, 104, 6140 .

36. Fox Ramos, A. E.; Alcover, C.; Evanno, L.; Maciuk, A.; Litaudon, M.; Duplais, C.; Bernadat, G.; Gallard, J. F.; Jullian, J. C.; Mouray, E.; Grellier, P.; Loiseau, P. M.; Pomel, S.; Poupon, E.; Champy, P.; Beniddir, M. A.; J. Nat. Prod. 2017, 80, 1007.

37. Mendes Resende, J. V.; de Sá, N. M. D.; de Oliveira, M. T. L.; Lopes, R. C.; Garrett, R.; Moreira Borges, R.; Phytochem. Lett. 2020, 36, 99.

38. Demarque, D. P.; Dusi, R. G.; de Sousa, F. D. M.; Grossi, S. M.; Silvério, M. R. S.; Lopes, N. P.; Espindola, L. S.; Sci. Rep. 2020, 10, 1051.

39. Aksenov, A. A.; Da Silva, R.; Knight, R.; Lopes, N. P.; Dorrestein, P. C.; Nat. Rev. Chem. 2017, $1,1$.

40. Gribkovskaia, I.; Halskau, Ø.; Laporte, G.; Networks 2007, 49, 199.

41. Guaratini, T.; Vessecchi, R.; Pinto, E.; Colepicolo, P.; Lopes, N. P.; J. Mass Spectrom. 2005, 40, 963.

42. Demarque, D. P.; Crotti, A. E. M.; Vessecchi, R.; Lopes, J. L. C.; Lopes, N. P.; Nat. Prod. Rep. 2016, 33, 432.

43. Alcoriza-Balaguer, M. I.; García-Cañaveras, J. C.; López, A.; Conde, I.; Juan, O.; Carretero, J.; Lahoz, A.; Anal. Chem. 2019, 91, 836.

44. Frank, A. M.; Bandeira, N.; Shen, Z.; Tanner, S.; Briggs, S. P.; Smith, R. D.; Pevzner, P. A.; J. Proteome Res. 2008, 7, 113.

45. Xie, H.-F.; Kong, Y.-S.; Li, R.-Z.; Nothias, L.-F.; Melnik, A. V.; Zhang, H.; Liu, L.-L.; An, T.-T.; Liu, R.; Yang, Z.; Ke, J.-P.; Zhang, P.; Bao, G.-H.; Xie, Z.-W.; Li, D.-X.; Wan, X.-C.; Dai, Q.-Y.; Zhang, L.; Zhao, M.; An, M.-Q.; Long, Y.-H.; Ling, T.-J.; J. Agric. Food Chem. 2020, 68 , 7995.

46. Nothias, L. F.; Nothias-Esposito, M.; da Silva, R.; Mingxun, W.; Protsyuk, I.; Zhang, Z.; Petras, D.; Sarvepalli, A.; Leyssen, P.; Touboul, D.; Costa, J.; Paolini, J.; Alexandrov, T.; Litaudon, M.; Dorrestein, P. C.; J. Nat. Prod. 2018, 81, 75 .

47. Gazolla, M. C.; Marques, L. M. M.; Silva, M. G.; Araújo, M. T. M. F.; Mendes, R. L.; Silva Almeida, J. R. G.; Vessecchi, R.; Lopes, N. P.; Rapid Commun. Mass Spectrom. 2020, 34.

48. Nothias-Esposito, M.; Nothias, L. F.; Da Silva, R. R.; Retailleau, P.; Zhang, Z.; Leyssen, P.; Roussi, F.; Touboul, D.; Paolini, J.; Dorrestein, P. C.; Litaudon, M.; J. Nat. Prod. 2019, 82, 1459.

49. Taboada, C.; Brunetti, A. E.; Pedron, F. N.; Carnevale Neto, F.; Estrin, D. A.; Bari, S. E.; Chemes, L. B.; Peporine Lopes, N.; Lagorio, M. G.; Faivovich, J.; Proc. Natl. Acad. Sci. 2017, 114, 3672.

50. Nothias, L. F.; Petras, D.; Schmid, R.; Dührkop, K.; Rainer, J.; Sarvepalli, A.; Protsyuk, I.; Ernst, M.; Tsugawa, H.; Fleischauer, M.; Aicheler, F.; Aksenov, A. A.; Alka, O.; Allard, P. M.; Barsch, A.; Cachet, X.; Caraballo-Rodriguez, A. M.; Da Silva, R. R.; Dang, T.; Garg, N.; 
Gauglitz, J. M.; Gurevich, A.; Isaac, G.; Jarmusch, A. K.; Kameník, Z.; Kang, K. Bin; Kessler, N.; Koester, I.; Korf, A.; Le Gouellec, A.; Ludwig, M.; Martin H, C.; McCall, L. I.; McSayles, J.; Meyer, S. W.; Mohimani, H.; Morsy, M.; Moyne, O.; Neumann, S.; Neuweger, H.; Nguyen, N. H.; Nothias-Esposito, M.; Paolini, J.; Phelan, V. V.; Pluskal, T.; Quinn, R. A.; Rogers, S.; Shrestha, B.; Tripathi, A.; van der Hooft, J. J. J.; Vargas, F.; Weldon, K. C.; Witting, M.; Yang, H.; Zhang, Z.; Zubeil, F.; Kohlbacher, O.; Böcker, S.; Alexandrov, T.; Bandeira, N.; Wang, M.; Dorrestein, P. C.; Nat. Methods 2020, 17, 905.

51. Pluskal, T.; Castillo, S.; Villar-Briones, A.; Orešič, M.; BMC Bioinformatics 2010, 11, 395.

52. Olivon, F.; Grelier, G.; Roussi, F.; Litaudon, M.; Touboul, D.; Anal. Chem. 2017, 89, 7836.

53. Röst, H. L.; Sachsenberg, T.; Aiche, S.; Bielow, C.; Weisser, H.; Aicheler, F.; Andreotti, S.; Ehrlich, H.-C.; Gutenbrunner, P.; Kenar, E.; Liang, X.; Nahnsen, S.; Nilse, L.; Pfeuffer, J.; Rosenberger, G.; Rurik, M.; Schmitt, W.; Veit, J.; Walzer, M.; Wojnar, D.; Wolski, W. E.; Schilling, O.; Choudhary, J.; Malmström, L.; Aebersold, R.; Reinert, K.; Kohlbacher, O.; Nat. Methods 2016, 13, 741.

54. Tsugawa, H.; Cajka, T.; Kind, T.; Ma, Y.; Higgins, B.; Ikeda, K.; Kanazawa, M.; Vandergheynst, J.; Fiehn, O.; Arita, M.; Nat. Methods 2015, 12, 523

55. Tautenhahn, R.; Patti, G. J.; Rinehart, D.; Siuzdak, G.; Anal. Chem. 2012, 84, 5035.

56. Hoffmann, N.; Rein, J.; Sachsenberg, T.; Hartler, J.; Haug, K.; Mayer, G.; Alka, O.; Dayalan, S.; Pearce, J. T. M.; Rocca-Serra, P.; Qi, D.; Eisenacher, M.; Perez-Riverol, Y.; Vizcaíno, J. A.; Salek, R. M.; Neumann, S.; Jones, A. R.; Anal. Chem. 2019, 91, 3302.

57. Katajamaa, M.; Miettinen, J.; Orešič, M.; Bioinformatics 2006, 22, 634.

58. Myers, O. D.; Sumner, S. J.; Li, S.; Barnes, S.; Du, X.; Anal. Chem. 2017, 89, 8696.

59. Jarmusch, A. K.; Wang, M.; Aceves, C. M.; Advani, R. S.; Aguirre, S.; Aksenov, A. A.; Aleti, G.; Aron, A. T.; Bauermeister, A.; Bolleddu, S.; Bouslimani, A.; Caraballo Rodriguez, A. M.; Chaar, R.; Coras, R.; Elijah, E. O.; Ernst, M.; Gauglitz, J. M.; Gentry, E. C.; Husband, M.; Jarmusch, S. A.; Jones, K. L.; Kamenik, Z.; Le Gouellec, A.; Lu, A.; McCall, L. I.; McPhail, K. L.; Meehan, M. J.; Melnik, A. V.; Menezes, R. C.; Montoya Giraldo, Y. A.; Nguyen, N. H.; Nothias, L. F.; NothiasEsposito, M.; Panitchpakdi, M.; Petras, D.; Quinn, R. A.; Sikora, N.; van der Hooft, J. J. J.; Vargas, F.; Vrbanac, A.; Weldon, K. C.; Knight, R.; Bandeira, N.; Dorrestein, P. C.; Nat. Methods 2020, 17, 901.

60. Aksenov, A.; Laponogov, I.; Zhang, Z.; Doran, S. L.; Belluomo, I.; Veselkov, D.; Bittremieux, W.; Nothias, L. F.; Nothias-Esposito, M.; Maloney, K.; Misra, B.; Melnik, A.; Jones, K.; Dorrestein, K.; Panitchpakdi, M.; Ernst, M.; van der Hooft, J.; Gonzalez, M.; Carazzone, C.; Amézquita, A.; Callewaert, C.; Morton, J.; Quinn, R.; Bouslimani, A.; Albarracín Orio, A.; Petras, D.; Smania, A.; Couvillion, S.; Burnet, M.; Nicora, C.; Zink, E.; Metz, T.; Artaev, V.; Humston-Fulmer, E.; Gregor, R.; Meijler, M.; Mizrahi, I.; Eyal, S.; Anderson, B.; Dutton, R.; Lugan, R.; Boulch, P. Le; Guitton, Y.; Prevost, S.; Poirier, A.; Dervilly, G.; Bizec, B. Le; Fait, A.; Persi, N. S.; Song, C.; Gashu, K.; Coras, R.; Guma, M.; Manasson, J.; Scher, J.; Barupal, D.; Alseekh, S.; Fernie, A.; Mirnezami, R.; Vasiliou, V.; Schmid, R.; Borisov, R.; Kulikova, L.; Knight, R.; Wang, M.; Hanna, G.; Dorrestein, P.; Veselkov, K.; 2020.

61. Smirnov, A.; Qiu, Y.; Jia, W.; Walker, D. I.; Jones, D. P.; Du, X.; Anal. Chem. 2019, 91, 9069.

62. Qiu, F.; Lei, Z.; Sumner, L. W.; Anal. Chim. Acta 2018, 1037, 316.

63. Remoroza, C. A.; Mak, T. D.; De Leoz, M. L. A.; Mirokhin, Y. A.; Stein, S. E.; Anal. Chem. 2018, 90, 8977.

64. Shannon, P.; Markiel, A.; Ozier, O.; Baliga, N. S.; Wang, J. T.; Ramage, D.; Amin, N.; Schwikowski, B.; Ideker, T.; Genome Res. 2003, 13, 2498.

65. Vargas, F.; Weldon, K. C.; Sikora, N.; Wang, M.; Zhang, Z.; Gentry, E. C.; Panitchpakdi, M. W.; Caraballo-Rodríguez, A. M.; Dorrestein, P. C.;
Jarmusch, A. K.; Rapid Commun. Mass Spectrom. 2020, 34.

66. Schymanski, E. L.; Ruttkies, C.; Krauss, M.; Brouard, C.; Kind, T.; Dührkop, K.; Allen, F.; Vaniya, A.; Verdegem, D.; Böcker, S.; Rousu, J.; Shen, H.; J. Cheminform. 2017, 1.

67. Ramos, A. E. F.; Pogam, P. Le; Alcover, C. F.; Otogo, E.; Nang, N.; Cauchie, G.; Hazni, H.; Awang, K.; Bréard, D.; Echavarren, A. M.; Frédérich, M.; Gaslonde, T.; Girardot, M.; Grougnet, R.; Kirillova, M. S.; Kritsanida, M.; Lémus, C.; Ray, A. Le; Lewin, G.; Litaudon, M.; Mambu, L.; Michel, S.; Miloserdov, F. M.; Muratore, M. E.; Richommepeniguel, P.; Roussi, F.; Sci. Data 2019, 6, 1.

68. Lei, Z.; Jing, L.; Qiu, F.; Zhang, H.; Huhman, D.; Zhou, Z.; Sumner, L. W.; 2015.

69. Nikolic, D.; Jones, M.; Dunn, L. S. and W.; Curr. Metabolomics 2017, 5,5 .

70. Horai, H.; Arita, M.; Kanaya, S.; Nihei, Y.; Nihei, Y.; Ikeda, T.; Suwa, K.; Ojima, Y.; Tanaka, K.; Tanaka, S.; Aoshima, K.; Oda, Y.; Kakazu, Y.; Kusano, M.; Tohge, T.; Matsuda, F.; Sawada, Y.; Hirai, M. Y.; Nakanishi. H.; Ikeda, K.; Akimoto, N.; Maoka, T.; Takahashi, H.; Ara, T.; Sakurai, N.; Suzuki, H.; Shibata, D.; Neumann, S.; Iida, T.; Tanaka, K.; Funatsu, K.; Matsuura, F.; Soga, T.; Taguchi, R.; Nishioka, T.; J. Mass Spec. 2010,45, 703.

71. Stravs, M. A.; Schymanski, E. L.; Singer, H. P.; Hollender, J.; J. Mass Spec. 2013, 48, 89 .

72. Hodgson, J.; Nat. Biotechnol. 2020, 38, 19.

73. Sumner, L. W.; Amberg, A. R.; Barrett, D.; Beale, Beger, R.; Daykin, C. A.; Fan, T. W.-M.; Fiehn, O.; Goodacre, R.; Griffin, J. L.; Hankemeier, T.; Hardy, N.; Harnly, J.; Higashi, R.; Kopka, J.; Lane, A. N.; Lindon, J. C.; Marriott, P.; Nicholls, A. W.; Reily, M. D.; Thaden, J. J.; Viant, M. R.; Metabolomics 2007, 3, 211.

74. da Silva, R. R.; Wang, M.; Nothias, L. F.; van der Hooft, J. J. J.; Caraballo-Rodríguez, A. M.; Fox, E.; Balunas, M. J.; Klassen, J. L.; Lopes, N. P.; Dorrestein, P. C.; PLoS Comput. Biol. 2018, 14, 1.

75. Da Silva, R. R.; Dorrestein, P. C.; Quinn, R. A.; Proc. Natl. Acad. Sci. U. S. A. 2015, 112, 12549.

76. Van Der Hooft, J. J. J.; Wandy, J.; Barrett, M. P.; Burgess, K. E. V.; Rogers, S.; Proc. Natl. Acad. Sci. U. S. A. 2016, 113, 13738

77. Wandy, J.; Zhu, Y.; Van Der Hooft, J. J. J.; Daly, R.; Barrett, M. P.; Rogers, S.; Bioinformatics 2018, 34, 317.

78. Ernst, M.; Kang, K. Bin; Caraballo-Rodríguez, A. M.; Nothias, L. F.; Wandy, J.; Wang, M.; Rogers, S.; Medema, M. H.; Dorrestein, P. C.; van der Hooft, J. J. J.; bioRxiv (2019), doi: 10.1101/654459.

79. Tripathi, A.; Vázquez-Baeza, Y.; Gauglitz, J. M.; Wang, M.; Dührkop, K.; Nothias-Esposito, M.; Acharya, D. D.; Ernst, M.; van der Hooft, J. J. J.; Zhu, Q.; McDonald, D.; Brejnrod, A. D.; Gonzalez, A.; Handelsman, J.; Fleischauer, M.; Ludwig, M.; Böcker, S.; Nothias, L. F.; Knight, R.; Dorrestein, P. C.; Nat. Chem. Biol. 2021, 17, 146.

80. Ruttkies, C.; Schymanski, E. L.; Wolf, S.; Hollender, J.; Neumann, S.; J. Cheminform. 2016, 8,1 .

81. Gerlich, M.; Neumann, S.; J. Mass Spectrom. 2013, 48, 291

82. Kang, K. Bin; Woo, S.; Ernst, M.; van der Hooft, J. J. J.; Nothias, L. F.; da Silva, R. R.; Dorrestein, P. C.; Sung, S. H.; Lee, M.; Phytochemistry 2020, 173.

83. Leão, T.; Wang, M.; Moss, N.; da Silva, R.; Sanders, J.; Nurk, S.; Gurevich, A.; Humphrey, G.; Reher, R.; Zhu, Q.; Belda-Ferre, P.; Glukhov, E.; Whitner, S.; Alexander, K. L.; Rex, R.; Pevzner, P.; Dorrestein, P. C.; Knight, R.; Bandeira, N.; Gerwick, W. H.; Gerwick, L.; Mar. Drugs 2021, 19.

84. Lee, J.; da Silva, R. R.; Jang, H. S.; Kim, H. W.; Kwon, Y. S.; Kim, J. H.; Yang, H.; Food Chem. 2019, 295, 368.

85. Blei, D. M.; Ng, A. Y.; Jordan, M. I.; J. Mach. Learn. Res. 2003, 3, 993.

86. Djoumbou Feunang, Y.; Eisner, R.; Knox, C.; Chepelev, L.; Hastings, J.; Owen, G.; Fahy, E.; Steinbeck, C.; Subramanian, S.; Bolton, E.; Greiner, R.; Wishart, D. S.; J. Cheminform. 2016, 8, 1. 
87. Di Ottavio, F.; Gauglitz, J. M.; Ernst, M.; Panitchpakdi, M. W.; Fanti, F.; Compagnone, D.; Dorrestein, P. C.; Sergi, M.; Food Chem. 2020, 313.

88. Dührkop, K.; Fleischauer, M.; Ludwig, M.; Aksenov, A. A.; Melnik, A. V.; Meusel, M.; Dorrestein, P. C.; Rousu, J.; Böcker, S.; Nat. Methods 2019, 16, 299.

89. Dührkop, K.; Shen, H.; Meusel, M.; Rousu, J.; Böcker, S.; Proc. Natl. Acad. Sci. U. S. A. 2015, 112, 12580.

90. Letunic, I.; Bork, P.; Nucleic Acids Res. 2019, 47, 256.

91. Bolyen, E.; Rideout, J. R.; Dillon, M. R.; Bokulich, N. A.; Abnet, C. C.; Al-Ghalith, G. A.; Alexander, H.; Alm, E. J.; Arumugam, M.; Asnicar, F.; Bai, Y.; Bisanz, J. E.; Bittinger, K.; Brejnrod, A.; Brislawn, C. J.; Brown, C. T.; Callahan, B. J.; Caraballo-Rodríguez, A. M.; Chase, J.; Cope, E. K.; Da Silva, R.; Diener, C.; Dorrestein, P. C.; Douglas, G. M.; Durall, D. M.; Duvallet, C.; Edwardson, C. F.; Ernst, M.; Estaki, M.; Fouquier, J.; Gauglitz, J. M.; Gibbons, S. M.; Gibson, D. L.; Gonzalez, A.; Gorlick, K.; Guo, J.; Hillmann, B.; Holmes, S.; Holste, H.; Huttenhower, C.; Huttley, G. A.; Janssen, S.; Jarmusch, A. K.; Jiang, L.; Kaehler, B. D.; Kang, K. Bin; Keefe, C. R.; Keim, P.; Kelley, S. T.; Knights, D.; Koester, I.; Kosciolek, T.; Kreps, J.; Langille, M. G. I.; Lee, J.; Ley, R.; Liu, Y. X.; Loftfield, E.; Lozupone, C.; Maher, M.; Marotz, C.; Martin, B. D.; McDonald, D.; McIver, L. J.; Melnik, A. V.; Metcalf, J. L.; Morgan, S. C.; Morton, J. T.; Naimey, A. T.; Navas-Molina, J. A.; Nothias, L. F.; Orchanian, S. B.; Pearson, T.; Peoples, S. L.; Petras, D.; Preuss, M. L.; Pruesse, E.; Rasmussen, L. B.; Rivers, A.; Robeson, M. S.; Rosenthal, P.; Segata, N.; Shaffer, M.; Shiffer, A.; Sinha, R.; Song, S. J.; Spear, J. R.; Swafford, A. D.; Thompson, L. R.; Torres, P. J.; Trinh, P.; Tripathi, A.; Turnbaugh, P. J.; Ul-Hasan, S.; van der Hooft, J. J. J.; Vargas, F.; Vázquez-Baeza, Y.; Vogtmann, E.; von Hippel, M.; Walters, W.; Wan, Y.; Wang, M.; Warren, J.; Weber, K. C.; Williamson, C. H. D.; Willis, A. D.; Xu, Z. Z.; Zaneveld, J. R.; Zhang, Y.; Zhu, Q.; Knight, R.; Caporaso, J. G.; Nat. Biotechnol. 2019, 37, 852.

92. Naman, C. B.; Rattan, R.; Nikoulina, S. E.; Lee, J.; Miller, B. W.; Moss, N. A.; Armstrong, L.; Boudreau, P. D.; Debonsi, H. M.; Valeriote, F. A.; Dorrestein, P. C.; Gerwick, W. H.; J. Nat. Prod. 2017, 80, 625.

93. Klein-Júnior, L. C.; Cretton, S.; Allard, P. M.; Genta-Jouve, G.; Passos, C. S.; Salton, J.; Bertelli, P.; Pupier, M.; Jeannerat, D.; Heyden, Y. Vander; Gasper, A. L.; Wolfender, J. L.; Christen, P.; Henriques, A. T.; J. Nat. Prod. 2017, 80, 3032.

94. Olivon, F.; Allard, P. M.; Koval, A.; Righi, D.; Genta-Jouve, G.; Neyts, J.; Apel, C.; Pannecouque, C.; Nothias, L. F.; Cachet, X.; Marcourt, L.; Roussi, F.; Katanaev, V. L.; Touboul, D.; Wolfender, J. L.; Litaudon, M.; ACS Chem. Biol. 2017, 12, 2644.

95. Caesar, L. K.; Kellogg, J. J.; Kvalheim, O. M.; Cech, R. A.; Cech, N. B.; Planta Med. 2018, 84, 721.

96. Hou, X. M.; Li, Y. Y.; Shi, Y. W.; Fang, Y. W.; Chao, R.; Gu, Y. C.; Wang, C. Y.; Shao, C. L.; J. Org. Chem. 2019, 84, 1228.

97. Velasco-Alzate, K. Y.; Bauermeister, A.; Tangerina, M. M. P.; Lotufo, T. M. C.; Ferreira, M. J. P.; Jimenez, P. C.; Padilla, G.; Lopes, N. P.; CostaLotufo, L. V.; Mar. Drugs 2019, 17, 671.

98. Bauermeister, A.; Pereira, F.; Grilo, I. R.; Godinho, C. C.; Paulino, M.; Almeida, V.; Gobbo-Neto, L.; Prieto-Davó, A.; Sobral, R. G.; Lopes, N. P.; Gaudêncio, S. P.; Environ. Microbiol. 2019, 21, 1099.

99. Floros, D. J.; Jensen, P. R.; Dorrestein, P. C.; Koyama, N.; Diego, S.; Jolla, L.; Diego, S.; Jolla, L.; Spectrometry, C. M.; Diego, S.; Jolla, L.; Metabolomics 2017, 12, 145.

100. Nothias, L. F.; Nothias-Esposito, M.; Da Silva, R.; Wang, M.; Protsyuk, I.; Zhang, Z.; Sarvepalli, A.; Leyssen, P.; Touboul, D.; Costa, J.; Paolini, J.; Alexandrov, T.; Litaudon, M.; Dorrestein, P. C.; J. Nat. Prod. 2018, 81,758 .

101. Crotti, A. E. M.; Vessecchi, R.; Lopes, J. L. C.; Lopes, N. P.; Quim. Nova 2006, 29, 287.

102. Braga, R. M.; Dourado, M. N.; Araújo, W. L.; Braz. J. Microbiol. 2016, 47,86
103. Brunetti, A. E.; Carnevale Neto, F.; Vera, M. C.; Taboada, C.; Pavarini, D. P.; Bauermeister, A.; Lopes, N. P.; Chem. Soc. Rev. 2018, 47, 1574.

104. Caraballo-Rodríguez, A. M.; Dorrestein, P. C.; Pupo, M. T.; Sci. Rep. 2017.

105. Vallet, M.; Vanbellingen, Q. P.; Fu, T.; Le Caer, J. P.; Della-Negra, S.; Touboul, D.; Duncan, K. R.; Nay, B.; Brunelle, A.; Prado, S.; J. Nat. Prod. 2017, 80, 2863.

106. Costa-Lotufo, L. V.; Carnevale-Neto, F.; Trindade-Silva, A. E.; Silva, R. R.; Silva, G. G. Z.; Wilke, D. V.; Pinto, F. C. L.; Sahm, B. D. B.; Jimenez, P. C.; Mendonça, J. N.; Lotufo, T. M. C.; Pessoa, O. D. L.; Lopes, N. P.; Chem. Commun. 2018, 54, 1952.

107. Hartmann, A. C.; Petras, D.; Quinn, R. A.; Protsyuk, I.; Archer, F. I.; Ransome, E.; Williams, G. J.; Bailey, B. A.; Vermeij, M. J. A.; Alexandrov, T.; Dorrestein, P. C.; Rohwer, F. L.; Proc. Natl. Acad. Sci. U. S. A. 2017, 114, 11685.

108. Silva, E.; da Graça, J. P.; Porto, C.; Martin do Prado, R.; HoffmannCampo, C. B.; Meyer, M. C.; de Oliveira Nunes, E.; Pilau, E. J.; Sci. Rep. 2020, 10, 1.

109. Taboada, C.; Brunetti, A. E.; Lyra, M. L.; Fitak, R. R.; Faigón Soverna, A.; Ron, S. R.; Lagorio, M. G.; Haddad, C. F. B.; Lopes, N. P.; Johnsen, S.; Faivovich, J.; Chemes, L. B.; Bari, S. E.; Proc. Natl. Acad. Sci. U. S. A. 2020, 117, 18574.

110. Kleigrewe, K.; Almaliti, J.; Tian, I. Y.; Kinnel, R. B.; Korobeynikov, A.; Monroe, E. A.; Duggan, B. M.; Marzo, V. Di; Sherman, D. H.; Dorrestein, P. C.; Gerwick, L.; Gerwick, W. H.; J. Nat. Prod. 2015, 78, 1671.

111. Maansson, M.; Vynne, N. G.; Klitgaard, A.; Nybo, J. L.; Melchiorsen, J.; Nguyen, D. D.; Sanchez, L. M.; Ziemert, N.; Dorrestein, P. C.; Andersen, M. R.; Grama, L.; mSystems 2016, 1,1

112. Parkinson, E. I.; Tryon, J. H.; Goering, A. W.; Ju, K. S.; McClure, R. A.; Kemball, J. D.; Zhukovsky, S.; Labeda, D. P.; Thomson, R. J.; Kelleher, N. L.; Metcalf, W. W.; ACS Chem. Biol. 2018, 13, 1029.

113. Klitgaard, A.; Nielsen, J. B.; Frandsen, R. J. N.; Andersen, M. R.; Nielsen, K. F.; Anal. Chem. 2015, 87, 6520.

114. Perez, C. E.; Crawford, J. M.; Biochemistry 2019, 58, 1131.

115. Silva-Junior, E. A.; Paludo, C. R.; Amaral, J. G.; Gallon, M. E.; GobboNeto, L.; Nascimento, F. S.; Lopes, N. P.; ACS Omega 2019, 4, 15208.

116. Rak Lee, S.; Schalk, F.; Schwitalla, J. W.; Benndorf, R.; Vollmers, J.; Kaster, A.-K.; de Beer, Z. W.; Park, M.; Ahn, M.-J.; Jung, W. H.; Beemelmanns, C.; Kim, K. H.; J. Nat. Prod. 2020, 83, 3102.

117. Conde-Martínez, N.; Bauermeister, A.; Pilon, A. C.; Lopes, N. P.; Tello, E.; Mar. Drugs 2019, 17.

118. Yue, Y.; Zhang, Q.; Wang, J.; Mar. Drugs 2019, 17, 395.

119. Bonneau, N.; Chen, G.; Lachkar, D.; Boufridi, A.; Gallard, J.-F.; Retailleau, P.; Petek, S.; Debitus, C.; Evanno, L.; Beniddir, M. A.; Poupon, E.; Chem. - A Eur. J. 2017, 23, 14454.

120. Zhu, G.; Hou, C.; Yuan, W.; Wang, Z.; Zhang, J.; Jiang, L.; Karthik, L.; Li, B.; Ren, B.; Lv, K.; Lu, W.; Cong, Z.; Dai, H.; Hsiang, T.; Zhang, L.; Liu, X.; Chem. Commun. 2020, 56, 10171.

121. A. Boya P., C.; Christian, M. H.; Fernández-Marín, H.; Gutiérrez, M.; Nat. Prod. Commun. 2019, 14, 1934578X1901400.

122. Gaudry, A.; Quirós, L.; Rutz, A.; Dounoue, M.; Kaiser, M.; David, B.; Marcourt, L.; Queiroz, E.; Wolfender, J.-L.; Allard, P.-M.; Resumo da $67^{\text {th }}$ International Congress and Annual Meeting of the Society for Medicinal Plant and Natural Product research (GA) in Cooperation with the French Society of Pharmacognosy AFERP, Innsbruck, Austria, 2019.

123. Chacon, D. S.; Torres, T. M.; da Silva, I. B.; de Araújo, T. F.; Roque, A. de A.; Pinheiro, F. A. S. D.; Selegato, D.; Pilon, A.; Reginaldo, F. P. S.; da Costa, C. T.; Vilasboa, J.; Freire, R. T.; Voigt, E. L.; Zuanazzi, J. A. S.; Libonati, R.; Rodrigues, J. A.; Santos, F. L. M.; Scortecci, K. C.; Lopes, N. P.; Ferreira, L. D. S.; dos Santos, L. V.; Cavalheiro, A. J.; Fett-Neto, A. G.; Giordani, R. B.; J. Adv. Res. 2021. 